\title{
Probabilistic Diffusion Tractography and Graph Theory Analysis Reveal Abnormal White Matter Structural Connectivity Networks in Drug-Naive Boys with Attention Deficit/Hyperactivity Disorder
}

\author{
Qingjiu Cao, ${ }^{1,2 *}$ Ni Shu, ${ }^{3 *}$ Li An,,${ }^{1,2}$ Peng Wang, ${ }^{1,2}$ Li Sun, ${ }^{1,2}$ Ming-Rui Xia, ${ }^{3}$ Jin-Hui Wang, ${ }^{3}$ Gao-Lang Gong, ${ }^{3}$ \\ Yu-Feng Zang, ${ }^{4,5}$ Yu-Feng Wang, ${ }^{1,2}$ and Yong $\mathrm{He}^{3}$ \\ ${ }^{1}$ Institute of Mental Health, Peking University, and ${ }^{2}$ Key Laboratory of Mental Health, Ministry of Health, Beijing 100191, China, ${ }^{3}$ State Key Laboratory of \\ Cognitive Neuroscience and Learning, Beijing Normal University, Beijing 100875, China, and ${ }^{4}$ Center for Cognition and Brain Disorders, Affiliated Hospital,

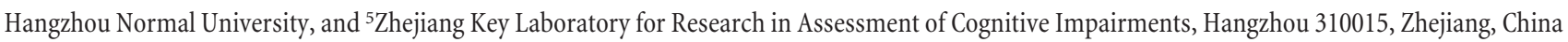

Attention-deficit/hyperactivity disorder (ADHD), which is characterized by core symptoms of inattention and hyperactivity/impulsivity, is one of the most common neurodevelopmental disorders of childhood. Neuroimaging studies have suggested that these behavioral disturbances are associated with abnormal functional connectivity among brain regions. However, the alterations in the structural connections that underlie these behavioral and functional deficits remain poorly understood. Here, we used diffusion magnetic resonance imaging and probabilistic tractography method to examine whole-brain white matter (WM) structural connectivity in 30 drugnaive boys with ADHD and 30 healthy controls. The WM networks of the human brain were constructed by estimating inter-regional connectivity probability. The topological properties of the resultant networks (e.g., small-world and network efficiency) were then analyzed using graph theoretical approaches. Nonparametric permutation tests were applied for between-group comparisons of these graphic metrics. We found that both the ADHD and control groups showed an efficient small-world organization in the whole-brain WM networks, suggesting a balance between structurally segregated and integrated connectivity patterns. However, relative to controls, patients with ADHD exhibited decreased global efficiency and increased shortest path length, with the most pronounced efficiency decreases in the left parietal, frontal, and occipital cortices. Intriguingly, the ADHD group showed decreased structural connectivity in the prefrontal-dominant circuitry and increased connectivity in the orbitofrontal-striatal circuitry, and these changes significantly correlated with the inattention and hyperactivity/impulsivity symptoms, respectively. The present study shows disrupted topological organization of large-scale WM networks in ADHD, extending our understanding of how structural disruptions of neuronal circuits underlie behavioral disturbances in patients with ADHD.

\section{Introduction}

Attention-deficit/hyperactivity disorder (ADHD), which is characterized by inattention and hyperactivity/impulsivity, is one of

Received Oct. 10, 2012; revised May 9, 2013; accepted May 17, 2013.

Author contributions: Q.C., N.S., Y.Z., Y.W., and Y.H. designed research; Q.C., N.S., L.A., P.W., L.S., and Y.W. performed research; N.S., M.X., J.W., G.G., and Y.H. contributed unpublished reagents/analytic tools; N.S. analyzed data; Q.C., N.S., Y.W., and Y.H. wrote the paper.

This work was supported by the Commonweal Sciences Foundation, Ministry of Health, China (Grant 2008 02073), the National Foundation, Ministry of Science and Technology, China (Grant 2007 BAl17B03), the National Science Fund for Distinguished Young Scholars Grant (Grant 81225012), the Natural Science Foundation of China (Grants 30970802, 31221003, 81030028, 81000633, 81000593 and 81020108022), and the Open Research Fund of the State Key Laboratory of Cognitive Neuroscience and Learning, Beijing Normal University, China. The authors thank the children and families who participated in the study.

${ }^{*}$ Q.C. and N.S. contributed equally to this work.

The authors declare no competing financial interests or potential conflicts of interest.

Correspondence should be addressed to either of the following: Dr. Yufeng Wang, Institute of Mental Health, Peking University, Beijing 100191, China, E-mail: wangyf@bjmu.edu.cn; or Dr. Yong He, State Key Laboratory of Cognitive Neuroscience and Learning, Beijing Normal University, Beijing 100875, China, E-mail: yong.he@bnu.edu.cn.

DOI:10.1523/JNEUROSCI.4793-12.2013

Copyright $\odot 2013$ the authors $\quad 0270-6474 / 13 / 3310676-12 \$ 15.00 / 0$ the most common childhood neuropsychiatric disorders. Neuroimaging studies suggest that these behavioral abnormalities could arise from aberrant brain connectivity (Konrad and Eickhoff, 2010). For instance, dysfunction in the dorsal frontoparietal networks that subserve cognitive control are closely related to the core symptoms of the inattention domain; the dysfunctions of the orbitofrontal cortex and ventral striatum structures that subserve the processes of reward and motivation play a central role in the core symptoms of hyperactivity/impulsivity (Durston et al., 2011; Liston et al., 2011). However, these studies mainly focused on disturbances in the functional circuits of ADHD; the alterations in the structural circuits that underlie the behavioral and functional deficits remain poorly understood.

Diffusion MRI is a promising noninvasive technique for assessing the microstructural characteristics of white matter (WM) tracts. Specifically, diffusion MRI tractography can reconstruct the major WM tracts faithful to the known WM anatomy, allowing mapping of the brain's structural connectivity in vivo (Basser et al., 2000). Using diffusion MRI, several groups have shown 
ADHD-associated disruption of structural connectivity in specific WM tracts, involving the prefrontal, parietal, occipital, and subcortical WM (Ashtari et al., 2005; Casey et al., 2007; Makris et al., 2008; Pavuluri et al., 2009). These findings provide crucial support for the hypothesis that structural connectivity is disrupted in ADHD.

Despite these advances in research, it remains unclear as to whether the topological organization of WM networks is abnormal in patients with ADHD. Recent work on brain connectivity has suggested that human whole-brain WM networks can be reconstructed using diffusion MRI deterministic or probabilistic tractography (Hagmann et al., 2008; Gong et al., 2009a,b; van den Heuvel and Sporns, 2011). Graph theory analysis revealed that the resultant WM networks exhibit many nontrivial topological properties, such as small worldness and highly connected hubs. Moreover, the topological organization of WM networks is disrupted under pathological conditions, for example, Alzheimer's disease (Lo et al., 2010), schizophrenia (van den Heuvel et al., 2010; Zalesky et al., 2011), and multiple sclerosis (Shu et al., 2011). Recently, three studies have used resting-state functional MRI (R-fMRI) to show disruption of topological properties in the whole-brain functional networks in ADHD (Wang et al., 2009a; Cocchi et al., 2012; Tomasi and Volkow, 2012). However, no study has reported ADHD-related alterations of the topological organization of WM networks.

Here, we used diffusion MRI probabilistic tractography and graph theory to investigate the topological organization of wholebrain WM networks in drug-naive boys with ADHD and in healthy comparison subjects. Previous studies have suggested that stimulants can significantly influence the brain's structure and function in ADHD (Bush et al., 2008; Shaw et al., 2009); by using drug-naive subjects in the present study, we can exclude the effects of stimulants. In the present study, we sought to determine (1) whether patients with ADHD would show disrupted topological efficiency and abnormal nodal/connectional properties in the whole-brain WM networks; and (2) if so, whether these topological changes would significantly correlate with the behavioral/ clinical characteristics of ADHD.

\section{Materials and Methods}

\section{Participants}

Thirty boys with ADHD ( $8-14$ years) and 30 healthy control boys ( $8-14$ years) participated in the study. None of the boys in the ADHD group had ever taken a stimulant or other medication to treat inattention problems. The boys in the group with ADHD were recruited from the outpatients at the Peking University Institute of Mental Health, and their ADHD diagnosis was made according to a semi-structured diagnostic interview, the Schedule for Affective Disorders and Schizophrenia for School-Age Children-Present and Lifetime Version [Diagnostic and Statistical Manual of Mental Disorders, Fourth Edition (DSM-IV) criteria; Kaufman et al., 1997]. The parents of the children in the ADHD group scored their children using the ADHD Rating Scale-IV (RS-IV), which contains all the inattention and hyperactivity/impulsivity symptoms of ADHD according to DSM-IV: "never" is rated as 1, "occasionally" is rated as 2, "often" is rated as 3, and "always" is rated as 4 (DuPaul et al., 1998). The exclusion criteria included the following: (1) left-handedness or ambidexterity, as assessed by the Chinese Handedness Inventory (Li, 1983); (2) a previous event involving head trauma with loss of consciousness; (3) a history of neurological illness, significant head trauma, or other severe diseases; (4) a history of emotional disorders, affective disorders, Tourette syndrome, or any other Axis I psychiatric disorder; (5) severe language development delay or communication problems, determined from clinical history, parental interview, and observation of the children; and (6) a full-scale intelligence quotient (IQ) under 80, as measured by the Wechsler Intelligence Scale for Chinese Children-Revised
Table 1. Demographic, clinical, and brain size properties of all participants

\begin{tabular}{lccc}
\hline & Control $(n=30)$ & $\operatorname{ADHD}(n=30)$ & $p$ value \\
\hline Age (years) & $10.3 \pm 1.6(8-14)$ & $10.3 \pm 1.9(8-14)$ & 0.94 \\
IQ & $121.7 \pm 14.0(84-144)$ & $107.1 \pm 14.4(85-142)$ & $<0.001^{* *}$ \\
Inattention scores & $17.5 \pm 3.9(9-26)$ & $27.5 \pm 3.9(20-35)$ & $<0.001^{* *}$ \\
Impulsivity scores & $15.6 \pm 3.9(9-24)$ & $23.9 \pm 10.0(12-59)$ & $<0.001^{* *}$ \\
Total intracranial volume (L) $1.40 \pm 0.1(1.18-1.62)$ & $1.34 \pm 0.1(1.13-1.52)$ & $0.044^{*}$ \\
\hline
\end{tabular}

All controls and ADHD patients were boys and were matched for age. Values are mean \pm SD (range). All variables were compared between groups with two-sample $t$ test. ${ }^{*} p<0.05$; ${ }^{* *} p<0.001$.

(Gong and Cai, 1993). Of the 30 boys with ADHD, 15 (50\%) met the criteria for combined type and $15(50 \%)$ met the criteria for predominantly inattentive-type. Notably, $11(37 \%)$ of the boys with ADHD also had oppositional defiant disorder, and none of the boys with ADHD had conduct disorder. The control boys were recruited from a local primary school. The exclusion criteria for the controls were the same as for the ADHD group except for the ADHD diagnosis. After a detailed explanation of the entire study procedure, all boys agreed to participate in the study and written informed consent was obtained from their parents or guardians. This study was approved by the Research Ethics Review Board of the Institute of Mental Health, Peking University, and by the Institutional Review Board of the State Key Laboratory of Cognitive Neuroscience and Learning, Beijing Normal University. Table 1 presents the demographic information and clinical characteristics of all the participants.

\section{Image acquisition}

MRI data were acquired using a Siemens TRIO 3T scanner in the Imaging Center for Brain Research, Beijing Normal University. Participants lay supine with their head snugly fixed by straps and foam pads to minimize head movement. T1-weighted, sagittal 3D magnetization-prepared rapid acquisition gradient echo sequences that covered the entire brain [128 slices, slice thickness $=1.33 \mathrm{~mm}$, repetition time $(\mathrm{TR})=2530 \mathrm{~ms}$, echo time $(\mathrm{TE})=3.39 \mathrm{~ms}$, inversion time $=1100 \mathrm{~ms}$, flip angle $=7^{\circ}$, acquisition matrix $=256 \times 256$, field of view $($ FOV $)=256 \times 256 \mathrm{~mm}^{2}$, average $=1]$ were acquired. Diffusion tensor images (DTIs) were acquired using a single-shot echoplanar imaging sequence with coverage of the whole brain $[49$ axial slices, slice thickness $=2.5 \mathrm{~mm}$ with no interslice gap, $\mathrm{TR}=7200 \mathrm{~ms}, \mathrm{TE}=104 \mathrm{~ms}$, flip angle $=90^{\circ}, 64$ diffusion directions with $b=1000 \mathrm{~s} / \mathrm{mm}^{2}$, and an additional image without diffusion weighting (i.e., $b=0 \mathrm{~s} / \mathrm{mm}^{2}$ ), acquisition matrix $=128 \times 128$, $\mathrm{FOV}=230 \times 230 \mathrm{~mm}^{2}$, average $\left.=1\right]$.

\section{Data preprocessing}

The data preprocessing included eddy current and motion artifact correction of DTI data, calculation of the diffusion tensor, and estimation of the probabilistic distribution of fiber orientations from each voxel. Briefly, eddy current distortions and motion artifacts in the DTI dataset were corrected by applying affine alignment of each diffusion-weighted image to the $b=0$ image. After this process, the diffusion tensor elements were estimated by solving the Stejskal and Tanner equation, and then the reconstructed tensor matrix was diagonalized to obtain three eigenvalues $\left(\lambda_{1}, \lambda_{2}, \lambda_{3}\right)$ and eigenvectors. The probabilistic distribution of fiber orientations from each voxel was estimated with a two-tensor model (Behrens et al., 2003, 2007). All these procedures were performed using the FMRIB Diffusion Toolbox (FSL, version 4.1; http://www.fmrib.ox.ac. $\mathrm{uk} / \mathrm{fsl})$.

\section{Network construction}

Nodes and edges are the two basic elements of a network. In the present study, we defined all of the network nodes and edges using the following procedures.

Network node definition. The procedure of defining the nodes has been previously described (Gong et al., 2009b) and was performed here using SPM8 (http://www.fil.ion.ucl.ac.uk/spm). Briefly, individual T1weighted images were coregistered to the $b=0$ images in the DTI space. The transformed $\mathrm{T} 1$ images were then nonlinearly transformed to the ICBM152 T1 template in the MNI space. The inverse transformations were used to warp the automated anatomical labeling (AAL) atlas 
Table 2. Cortical and subcortical regions of interest defined in the study

\begin{tabular}{|c|c|c|c|c|c|}
\hline Index & Regions & Abbreviation & Index & Regions & $\overline{\text { Abbreviation }}$ \\
\hline$(1,2)$ & Precental gyrus & PreCG & $(47,48)$ & Lingual gyrus & LING \\
\hline$(3,4)$ & Superior frontal gyrus, dorsolateral & SFGdor & $(49,50)$ & Superior occipital gyrus & SOG \\
\hline$(5,6)$ & Superior frontal gyrus, orbital part & ORBsup & $(51,52)$ & Middle occipital gyrus & MOG \\
\hline$(7,8)$ & Middle frontal gyrus & MFG & $(53,54)$ & Inferior occipital gyrus & IOG \\
\hline$(9,10)$ & Middle frontal gyrus, orbital part & ORBmid & $(55,56)$ & Fusiform gyrus & FFG \\
\hline$(11,12)$ & Inferior frontal gyrus, opercular part & IFGoperc & $(57,58)$ & Postcentral gyrus & PoCG \\
\hline$(13,14)$ & Inferior frontal gyrus, triangular part & IFGtriang & $(59,60)$ & Superior parietal gyrus & SPG \\
\hline$(15,16)$ & Inferior frontal gyrus, orbital part & ORBinf & $(61,62)$ & Inferior parietal, but supramarginal and angular gyri & IPL \\
\hline$(17,18)$ & Rolandic operculum & ROL & $(63,64)$ & Supramarginal gyrus & SMG \\
\hline$(19,20)$ & Supplementary motor area & SMA & $(65,66)$ & Angular gyrus & ANG \\
\hline$(21,22)$ & Olfactory cortex & OLF & $(67,68)$ & Precuneus & PCUN \\
\hline$(23,24)$ & Superior frontal gyrus, medial & SFGmed & $(69,70)$ & Paracentral lobule & $\mathrm{PCL}$ \\
\hline$(25,26)$ & Superior frontal gyrus, medial orbital & ORBsupmed & $(71,72)$ & Caudate nucleus & CAU \\
\hline$(27,28)$ & Gyrus rectus & REC & $(73,74)$ & Lenticular nucleus, putamen & PUT \\
\hline$(29,30)$ & Insula & INS & $(75,76)$ & Lenticular nucleus, pallidum & PAL \\
\hline$(31,32)$ & Anterior cingulate and paracingulate gyri & ACG & $(77,78)$ & Thalamus & THA \\
\hline$(33,34)$ & Median cingulate and paracingulate gyri & DCG & $(79,80)$ & Heschl gyrus & HES \\
\hline$(35,36)$ & Posterior cingulate gyrus & PCG & $(81,82)$ & Superior temporal gyrus & STG \\
\hline$(37,38)$ & Hippocampus & HIP & $(83,84)$ & Temporal pole: superior temporal gyrus & TPOsup \\
\hline$(39,40)$ & Parahippocampal gyrus & PHG & $(85,86)$ & Middle temporal gyrus & MTG \\
\hline$(41,42)$ & Amygdala & AMYG & $(87,88)$ & Temporal pole: middle temporal gyrus & TP0mid \\
\hline$(43,44)$ & Calcarine fissure and surrounding cortex & CAL & $(89,90)$ & Inferior temporal gyrus & ITG \\
\hline$(45,46)$ & Cuneus & CUN & & & \\
\hline
\end{tabular}

The regions are listed in terms of a prior template of an AAL atlas (Tzourio-Mazoyer et al., 2002).

(Tzourio-Mazoyer et al., 2002) from the MNI space to the DTI native space. Discrete labeling values were preserved by the use of a nearestneighbor interpolation method. Using this procedure, we obtained 90 cortical and subcortical regions (45 for each hemisphere; Table 2), each representing a node of the network (Fig. 1).

WM tractography. To reconstruct the whole-brain WM tracts, we performed probabilistic tractography using the FMRIB Diffusion Toolbox (FSL, version 4.1; http://www.fmrib.ox.ac.uk/fsl). The probabilistic tractography was applied by sampling 5000 streamline fibers per voxel. For each sampled fiber, a sample direction was first drawn from the local direction distribution at the seed voxel, then we moved $0.5 \mathrm{~mm}$ in the sample direction to a new position, and finally, a new sample direction from the local distribution was obtained at this new position. For a seed region, $5000 \times n$ fibers were sampled; $n$ is the number of voxels in the region. The number of fibers passing through a given region divided by $5000 \times n$ is calculated as the connectivity probability from the seed region to the given region.

In the present study, each brain region was selected as the seed region, and its connectivity probability to each of the other 89 regions was calculated. Notably, the probability from $i$ to $j$ is not necessarily equivalent to the probability from $j$ to $i$ because the tractography is dependent on the seeding location. However, these two probabilities are highly correlated across the brain regions for all subjects (all Pearson $r>0.97, p<10^{-20}$ ). Thus, we defined the unidirectional connectivity probability $P_{i j}$ between region $i$ and region $j$ by averaging these two probabilities. For each subject, the estimation of the local connectivity distribution took $\sim 24 \mathrm{~h}$, and the probabilistic tractography for the 90 seed regions took $\sim 70 \mathrm{~h}$ using a single-core CPU. In the present study, all the processing steps were performed in parallel using an SGE-capable system with 60 processor cores.

Network edge definition. To define the network edges, we computed $w_{i j}=P_{i j}$ as the weight between brain regions $i$ and $j$. For each subject, a $90 \times 90$ symmetric weighted network was constructed. To remove spurious connections, we applied a threshold range between 0.01 and 0.1 at intervals of 0.0025 . Specifically, two brain regions were considered unconnected if the mean connectivity probability across the subjects was $>2$ SDs below a given threshold [e.g., mean $\left(P_{i j}\right)+2 \mathrm{SD}\left(P_{i j}\right)<$ threshold]. For a given threshold value, this scheme leads to the same number and position of the connections across subjects. Under the threshold, the corresponding network sparsities range from $6 \%$ to $24 \%$. The largest component sizes of individual networks remained at 90 across the sparsity range.

\section{Network analysis}

For the weighted WM networks at each threshold, we calculated both global and regional network metrics. The global metrics included: network strength $\left(S_{p}\right)$, global efficiency $\left(E_{\text {glob }}\right)$, local efficiency $\left(E_{\text {loc }}\right)$, shortest path length $\left(L_{p}\right)$, clustering coefficient $\left(C_{p}\right)$, and small-world parameters ( $\lambda$ and $\gamma$; Rubinov and Sporns, 2010). For regional characteristics, we considered the nodal efficiency metric (Achard and Bullmore, 2007). Furthermore, we calculated the area under the curve (AUC) for each network metric, which provides a summarized scalar for topological organization of brain networks independent of a single threshold selection. The integrated AUC metric has been applied in previous studies of brain networks (Achard et al., 2006; Gong et al., 2009a; He et al., 2009). To further localize specific region pairs in which WM connectivity was altered in patients with ADHD, we used a network-based statistic (NBS) approach (Zalesky et al., 2010a). All network analysis was performed using the GRETNA software (http://www.nitrc.org/projects/ gretna/). The definitions of these network properties are briefly described below.

Network strength. For a network (graph) $G$ with $N$ nodes and $K$ edges, we calculated the strength of $G$ as follows:

$$
S_{p}(G)=\frac{1}{N} \sum_{i \in G} S(i),
$$

where $S(i)$ is the sum of the edge weights $w_{i j}$ linking to node $i$. The strength of a network is the average of the strengths across all of the nodes in the network.

Small-world properties. Small-world network parameters $\left(C_{p}\right.$ and $\left.L_{p}\right)$ were originally proposed by Watts and Strogatz (1998). In this study, we investigated the small-world properties of the weighted brain networks. The weighted clustering coefficient of a node $i, C(i)$, which was defined as the likelihood that the neighborhoods were connected with each other (Onnela et al., 2005), is expressed as follows:

$$
C(i)=\frac{2}{k_{i}\left(k_{i}-1\right)} \sum_{j, k}\left(\bar{w}_{i j} \bar{w}_{j k} \bar{w}_{k i}\right)^{1 / 3},
$$

where $k_{i}$ is the degree of node $i$, and $\bar{w}$ is the weight, which is scaled by the mean of all weights to control each participant's cost at the same level. The clustering coefficient is zero, $C(i)=0$, if the nodes are isolated or 
A T1 anatomical parcellation
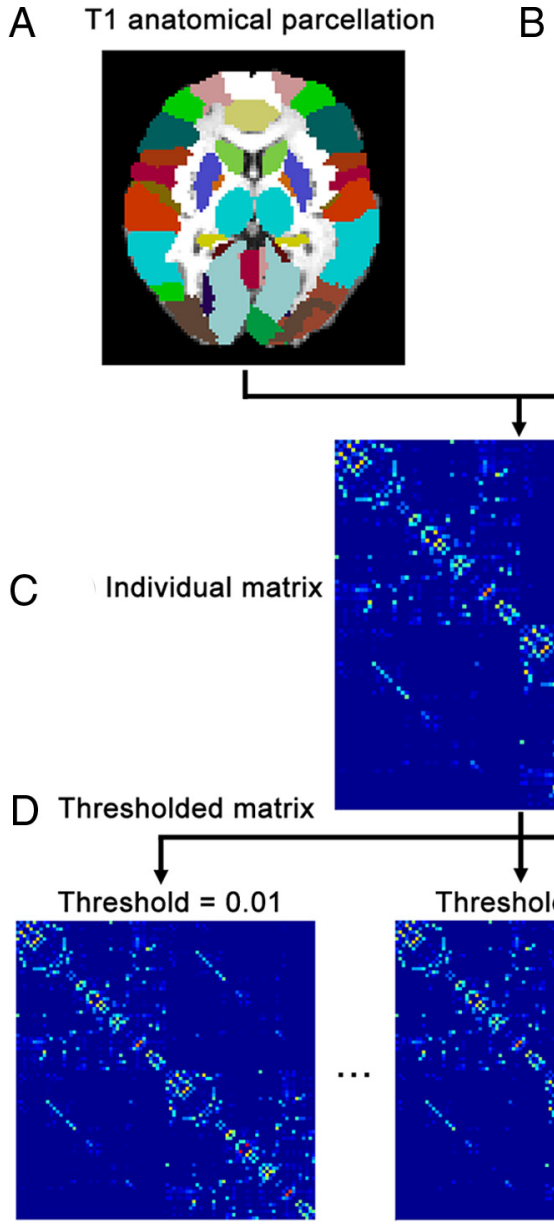

DTI probabilistic tractography
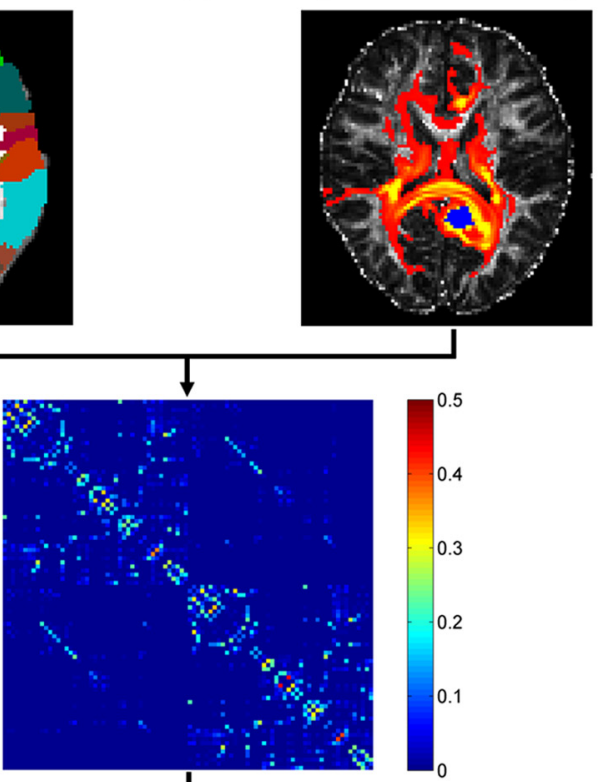

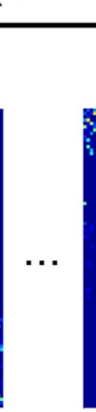

Threshold $=0.05$
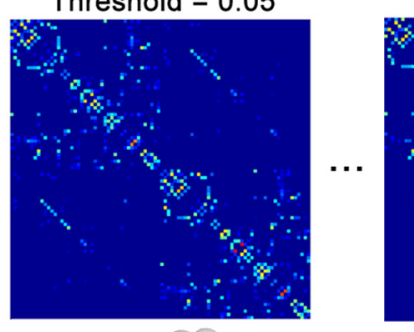

\section{Threshold $=0.1$}
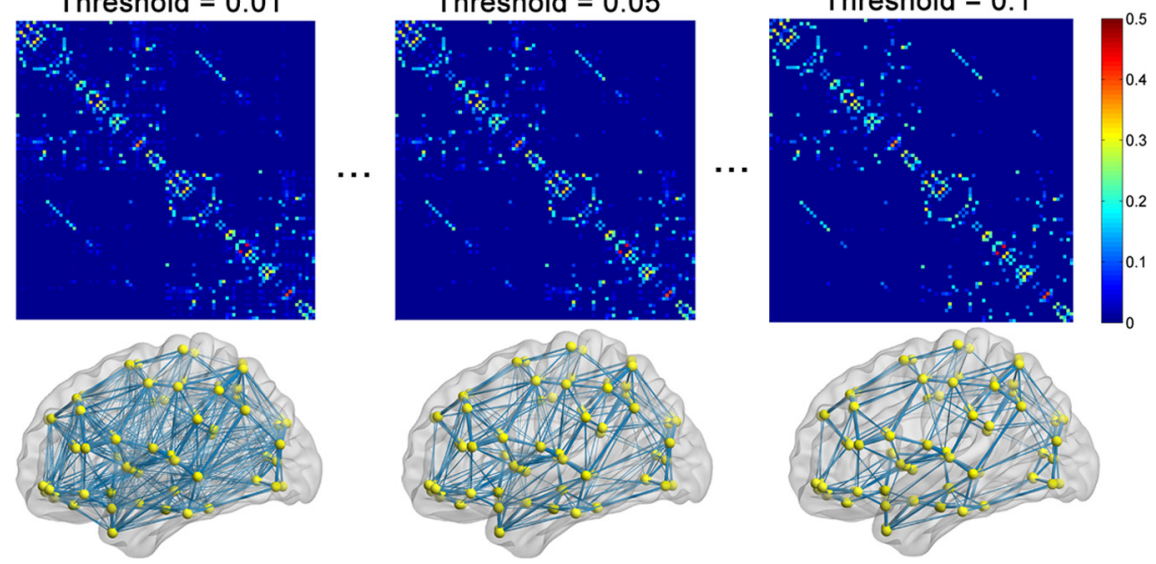

Figure 1. A flowchart for the construction of the WM structural network by diffusion MRI data. $\boldsymbol{A}$, Individual T1-weighted images were used to segment the brain into 90 cortical and subcortical regions according to the AAL template (see Materials and Methods). $\boldsymbol{B}$, For each region, the connectivity probability with other brain regions was estimated using diffusion MRI tractography. The yellow to red colors represent the resulting probability (yellow $>$ red) from the left precuneus (marked as blue) to the other voxels. C, The individual structural connectivity matrix was created by considering the connection probability between two regions. $\boldsymbol{D}$, The individual matrix was thresholded under a range of probability values $(0.01 \sim 0.1$, which corresponds to a sparsity range of $6-24 \%)$, and the resultant WM networks were presented in anatomical space. The nodes are located according to their centroid stereotaxic coordinates. For more details, see Materials and Methods.

have just one connection (i.e., $k_{i}=0$ or $k_{i}=1$ ). The clustering coefficient $C_{p}$ of a network is the average of the clustering coefficient over all nodes, which indicates the extent of local interconnectivity or cliquishness in a network (Watts and Strogatz, 1998).

The path length between any pair of nodes (e.g., node $i$ and node $j$ ) is defined as the sum of the edge lengths along this path. For weighted networks, the length of each edge was assigned by computing the reciprocal of the edge weight, $1 / w_{i j}$. The shortest path length $L_{i j}$, is defined as the length of the path for node $i$ and node $j$ with the shortest length. The shortest path length of $G$ is computed as follows:

$$
L_{p}(G)=\frac{1}{N(N-1)} \sum_{i \neq j \in G} L_{i j},
$$

which quantifies the ability for information to be propagated in parallel.
To examine the small-world properties, the $C_{p}$ and $L_{p}$ of the brain networks were compared with those of random networks. In this study, we generated 100 matched random networks, which had the same number of nodes and edges, and degree distribution as the real networks (Maslov and Sneppen, 2002). Of note, we retained the weight of each edge during the randomization procedure such that the weight distribution of the network was preserved. Furthermore, we computed the normalized $C_{p}$, $\gamma=C_{p}^{\text {real }} / C_{p}^{\text {rand }}$, and the normalized $L_{p}, \lambda=$ $L_{p}^{\text {real }} / L_{p}^{\text {rand }}$, where $C_{p}^{\text {rand }}$ and $L_{p}^{\text {rand }}$ are the mean $C_{p}$ and the mean $L_{p}$ of 100 matched random networks. A real network would be considered small world if $\gamma>1$ and $\lambda \approx 1$ (Watts and Strogatz, 1998). In other words, a small-world network has not only the higher local interconnectivity but also the approximately equivalent shortest path length compared with the random networks.

Network efficiency. The global efficiency of $G$ measures the global efficiency of the parallel information transfer in the network (Latora and Marchiori, 2001), which can be computed as follows:

$$
E_{\mathrm{glob}}(G)=\frac{1}{N(N-1)} \sum_{i \neq j \in G} \frac{1}{L_{i j}},
$$

where $L_{i j}$ is the shortest path length between node $i$ and node $j$ in $G$.

The local efficiency of $G$ reveals how much the network is fault tolerant, showing how efficient the communication is among the first neighbors of node $i$ when it is removed. The local efficiency of a graph is defined as follows:

$$
E_{\mathrm{loc}}(G)=\frac{1}{N} \sum_{i \in G} E_{\mathrm{glob}}\left(G_{i}\right),
$$

where $G_{i}$ denotes the subgraph composed of the nearest neighbors of node $i$.

Regional nodal characteristics. To determine regional nodal characteristics of the WM networks, we computed the regional efficiency $E_{\text {nodal }}(i)$ (Achard and Bullmore, 2007):

$$
E_{\text {nodal }}(i)=\frac{1}{N-1} \sum_{i \neq j \in G} \frac{1}{L_{i j}}
$$

where $L_{i j}$ is the shortest path length between node $i$ and node $j$ in $G$. $E_{\text {nodal }}(i)$ measures the average shortest path length between a given node $i$ and all of the other nodes in the network. Node $i$ was considered a brain hub if $E_{\text {nodal }}(i)$ was at least $1 \mathrm{SD}$ greater than the average nodal efficiency of the network (i.e., $E_{\text {nodal }}(i)>$ mean $+S D$ ).

NBS analysis. To localize specific pairs of brain regions in which structural connectivity was altered in $\mathrm{ADHD}$, we used a recently developed NBS approach (Zalesky et al., 2010a). Briefly, we first detected significantly nonzero connections within groups, which are the same across subjects. The NBS approach was then conducted within the connections as follows. A primary threshold $(p=0.01)$ was first applied to the $t$ statistic (two-sample one-tailed $t$ tests) computed for each link to define a set of suprathreshold links, among which any connected components and their size (number of links) were then determined. To estimate the significance for each component, the null distribution of connected component size was empirically derived using a nonparametric permutation 
approach (10,000 permutations). For each permutation, all subjects were randomly reallocated into two groups, and the $t$ statistic (two-sample one-tailed $t$ test) was computed independently for each link. Then, the threshold ( $p=0.05$ ) was used to generate suprathreshold links among which the maximal connected component size was recorded. Finally, for a connected component of size $M$ found in a right grouping of controls and patients, the corrected $p$ value was determined by finding the proportion of the 10,000 permutations for which the maximal connected component was larger than $M$. Notably, several previous diffusion MRI studies have suggested significant associations of age, IQ, and brain size with the topological properties of whole-brain WM networks (Gong et al., 2009a; Li et al., 2009; Yan et al., 2011). Thus, the effects of age, IQ, and brain size were removed by a regression analysis before the statistical analysis of the connections (see Statistical analysis). This NBS method has recently been used to identify abnormal brain connectivity circuitry in schizophrenia (Zalesky et al., 2011) and depression (Zhang et al., 2011; Bai et al., 2012). For a detailed description, see Zalesky et al. (2010a).

\section{Statistical analysis}

Between-group differences. Two-sample $t$ tests were used to test the between-group differences in age, IQ, brain size, and scores of ADHD symptom severity. To determine the statistical significance of group differences in network metrics, a nonparametric permutation method $(10,000$ permutations) was used (He et al., 2008). Briefly, for each network metric, we first computed the between-group difference in the mean AUC value. To test the null hypothesis that the observed group difference could occur by chance, we then randomly reallocated all the AUC values into one or the other of the two groups and recomputed the difference in the mean AUC values between the randomized groups. This randomization procedure was repeated 10,000 times, and the 95th percentile points of these distributions were used as the critical values for a one-tailed test of the null hypothesis with a probability of type I error of 0.05 . Before the permutation tests, the effects of age, IQ, and brain size were removed by the following multiple linear regression analysis.

$$
Y=\beta_{0}+\beta_{1} \times \text { age }+\beta_{2} \times \text { IQ }+\beta_{3} \times \text { brain size, }
$$

where $Y$ was a dependent variable indicating each network metric and the independent variables included age, IQ, and brain size. Likewise, permutation tests $(10,000$ permutations) were used to determine the significance levels of altered components in the NBS analysis.

Relationships between network metrics and clinical variables. For those network metrics with significant group differences, we examined the relationships between the metrics and the clinical variables in the ADHD group by performing multiple linear regression analyses (dependent variables: network metrics; independent variables: inattention or hyperactivity/impulsivity score measured by ADHD RS-IV). Age, IQ, and brain size were also treated as confounding covariates.

\section{Validation analysis}

Pediatric template. In the present study, we used the ICBM152 T1 template for spatial normalization. Such an adult template could lead to the misregistration of the images in the pediatric population. To assess whether our main results were affected by the use of the adult template, we used a pediatric T1 template (7.5-13.5 years of age; Fonov et al., 2011) for imaging normalization. We then repeated the whole-brain network construction and analysis procedures, including the WM tractography, network edge definition, and graphic analysis, followed by the statistical comparisons between the two groups. Additionally, for each network metric derived from two templates, we also computed the correlations across subjects for each group.

High-resolution brain network analysis. Several studies have suggested that graphic metrics of whole-brain networks are dependent on the resolution of the network (i.e., network size) (Wang et al., 2009b; Zalesky et al., 2010b). In addition to the coarse parcellation scheme using 90 nodes, we also used a high-resolution ( 1000 parcels) parcellation (H-1024) by randomly subdividing the AAL atlas into 1024 regions with equal size (Hagmann et al., 2008; Zalesky et al., 2010b). Similar to the low- resolution AAL networks, the connectivity probability between regions was defined as the weight of the network edge, which results in a symmetric $1024 \times 1024$ matrix for each participant. The sparsities of H-1024 networks under probability thresholds of $0.01-0.1$ (at intervals of 0.005 ) ranged from $1.6 \%$ to $8.4 \%$. Then, we repeated all the network analyses and statistical comparisons to determine the between-group differences in the graphic metrics. Probabilistic tracking of 1024 seed regions took $\sim 200 \mathrm{~h}$ per subject using a single-core CPU.

\section{Results}

There was no significant difference in age between the ADHD and control groups $(p>0.9)$. However, patients with ADHD exhibited significantly lower IQ scores $(p<0.001)$, higher inattention scores $(p<0.001)$, and higher hyperactivity/impulsivity scores $(p<0.001)$. Additionally, we observed that the boys with ADHD had a significantly smaller brain size ( $p=0.044)$ compared with the controls (Table 1).

\section{Alterations in the global properties of WM networks in ADHD}

Both the ADHD patients and controls showed a small-world organization of WM networks characterized by $\gamma>1$ and $\lambda \approx 1$ (Fig. 2). However, compared with controls, the ADHD patients had significantly decreased $E_{\text {glob }}$ and increased $L_{p}$ and $\lambda$ in the WM networks over a wide range of thresholds (Fig. 2). Additionally, we observed increased $E_{\mathrm{loc}}$ and $C_{p}$ in the ADHD networks over several thresholds (Fig. 2). Moreover, the ADHD patients showed significantly decreased AUC values of $E_{\text {glob }}(p=0.038)$, increased $L_{p}(p=0.029)$ and increased $\lambda(p=0.046)$ in the WM networks (Table 3 ). There were no significant differences between groups in the other network metrics (Fig. 2; Table 3).

\section{Alterations in the regional properties of WM networks in ADHD}

First, we identified the hub regions of the WM networks for each group. The nodes were considered brain hubs if their nodal efficiencies were at least 1 SD greater than the average nodal efficiency of the network. We found that the ADHD and control groups showed highly similar hub distributions, with core regions mainly in the medial frontal and parietal cortices (Fig. $3 A, B$; Table 4). This finding is largely consistent with previous WM network studies in healthy adults (Hagmann et al., 2008; Gong et al., 2009a,b; van den Heuvel and Sporns, 2011). Further statistical analysis revealed that patients with ADHD had reduced nodal efficiency in the left parietal (supramarginal gyrus, inferior parietal cortex, postcentral gyrus, and angular gyrus), left frontal (orbital middle frontal gyrus), and left occipital cortices (lingual gyrus; $p<0.05$, uncorrected; Fig. $3 C$; Table 5).

\section{Decreased prefrontal-dominant component in ADHD}

Using the NBS analysis, we found that ADHD patients showed significantly decreased WM connections in the left prefrontaldominant component under most of the thresholds (mainly composed of the left dorsolateral parts of the superior frontal gyrus, the precentral gyrus and the orbital parts of the superior and middle frontal gyri; $p$ values $<0.05$ at multiple probability thresholds, corrected; Fig. 4A). The strengths, percentages, and frequencies of these decreased WM connections across thresholds are shown in Table 6.

\section{Increased orbitofrontal-striatal component in ADHD}

Using the NBS analysis, we also showed that ADHD patients exhibited significant (or trends toward significant) increases in 

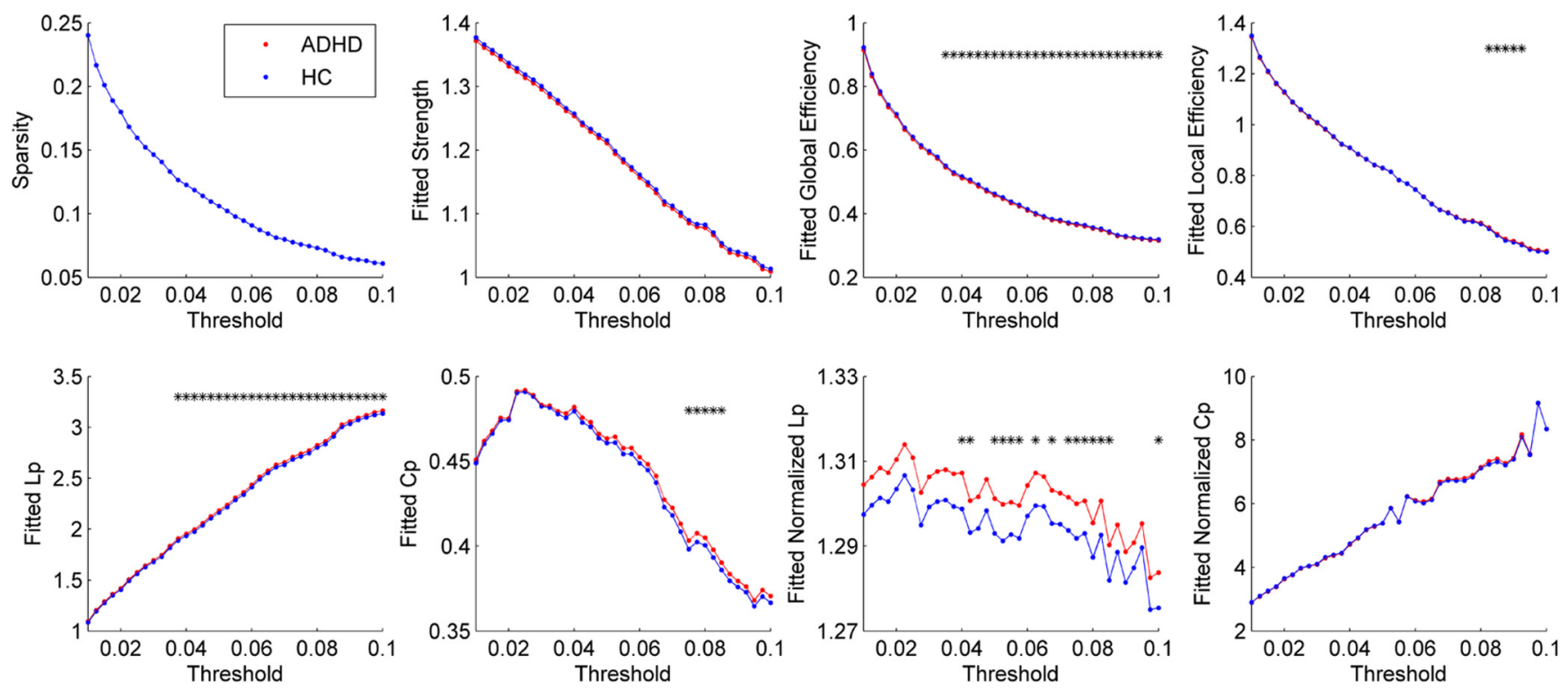

Figure 2. Differences in topological properties of WM structural networks between ADHD patients and controls. Global metrics of WM structural networks were quantified in controls and ADHD patients with different probability thresholds. Data points marked with a star indicate a significant group difference $(p<0.05)$ in the global network metric under the threshold. Both ADHD patients and controls showed a small-world organization of WM networks characterized by a $\gamma>1$ and $\lambda \approx 1$. However, compared with controls, ADHD patients had significantly decreased $E_{\text {glob, }}$ increased absolute $L_{p}$, and $\lambda$ in the WM networks for most of thresholds considered. $H C$, Healthy controls. The fitted values mean the residuals of original values of network metrics after removing the effects of age, $I Q$, and brain size.

Table 3. Group comparisons of AUC values of global network properties

\begin{tabular}{llllllll}
\hline & $S_{p}$ & $E_{\text {glob }}$ & $E_{\text {loc }}$ & $L_{p}$ & $C_{p}$ & $\lambda$ \\
\hline Control & $42.76 \pm 1.37$ & $17.41 \pm 0.24$ & $29.18 \pm 0.25$ & $80.90 \pm 1.06$ & $15.81 \pm 0.28$ & $46.60 \pm 0.45$ \\
ADHD & $42.60 \pm 1.82$ & $17.26 \pm 0.23$ & $29.19 \pm 0.24$ & $81.62 \pm 1.01$ & $15.92 \pm 0.24$ & $46.86 \pm 0.45$ & $204.12 \pm 2.52$ \\
$p$ value & 0.39 & $0.038^{*}$ & 0.45 & $0.029^{*}$ & 0.13 & $0.046^{*}$ & 0.30 \\
\hline
\end{tabular}

Permutation tests were used to determine the differences in the global network properties between groups (see Materials and Methods). Values were the fitted AUC values (mean \pm SD) of global network properties in each group. ${ }^{*} p<0.05$.

WM connections in the orbitofrontal-striatal component under most of the thresholds (mainly composed of the striatum structures including the caudate and putamen and the medial orbital parts of the superior frontal gyrus; $p=0.015$ at the threshold of 0.01 and $p<0.10$ at several other thresholds, corrected; Fig. $4 B$ ). The strengths, percentages, and frequencies of these increased WM connections across thresholds are shown in Table 6.

\section{Relationships between network metrics and ADHD symptoms}

Under multiple probability thresholds, we found that the mean NBS connectivity strength of the decreased prefrontal-dominant component was negatively correlated with the inattention score in the ADHD patients $(p<0.035$; Fig. $4 A)$. At the probability threshold $=0.01$ ( parsity $=24 \%)$, the mean NBS connectivity strength of the increased orbitofrontal-striatal component was positively correlated with the hyperactivity/impulsivity score in ADHD patients $(p=0.032$; Fig. $4 B)$. There were no significant correlations between the other network metrics and ADHD symptoms.

\section{Validation results}

The effects of the pediatric template

While using the pediatric T1 template for spatial normalization, the results were highly consistent with those obtained from the adult ICBM152 template. Compared with the controls, the ADHD patients showed significantly decreased AUC values of $E_{\text {glob }}(p=0.043)$ and significantly increased $L_{p}(p=0.031)$ and $\lambda$ $(p=0.048)$; there were no significant group differences in the other network metrics. Reduced nodal efficiencies in the ADHD patients were primarily located in the left parietal cortex (supramarginal gyrus, inferior parietal cortex, postcentral gyrus, angular gyrus, and paracentral lobule) and left insula $(p<0.05$, uncorrected). NBS analysis revealed a significantly decreased prefrontal-dominant component and an increased orbitofrontalstriatal component in the ADHD group ( $p<0.05$, corrected). Additionally, for each group we also observed strong correlations in both the global network properties $\left(S_{p}\right.$ : ADHD, $r=0.97$ and control, $r=0.97$; $E_{\text {glob }}$ ADHD, $r=0.78$ and control, $r=0.82$; $E_{\text {loc }}:$ ADHD, $r=0.84$ and control, $r=0.86 ; L_{p}: \mathrm{ADHD}, r=0.77$ and control, $r=0.79$; $C_{p}$ : ADHD, $r=0.90$ and control, $r=0.94$; all $p$ values $<10^{-5}$ ) and the nodal efficiency (ADHD, mean $r=$ 0.87 ; control, mean $r=0.86$; all $p$ values $<10^{-5}$ ) between the two templates across the subjects.

\section{The effects of high-resolution parcellation}

In contrast to the results of low-resolution AAL networks, we found no significant between-group differences in the global network properties for the $\mathrm{H}-1024$ networks $(p>0.05)$. For nodal analysis, the regions with decreased efficiency in ADHD were located in the left parietal cortex (paracentral lobule, inferior parietal cortex, supramarginal gyrus, and postcentral gyrus) and the right supplementary motor area, while increased efficiencies were mainly distributed in the prefrontal cortex (bilateral medial superior frontal gyri, right middle and inferior frontal gyri, right anterior cingulate gyrus, and right precentral gyrus), the left 
parahippocampus gyrus, and the right superior occipital gyrus $(p<0.05$, uncorrected; Fig. $5 A$ ). Using NBS analysis, both significantly decreased and increased components across thresholds were identified ( $p$ values $<0.05$, corrected). The decreased component was mainly involved in the WM connections of the left dorsal prefrontal cortex, the left precentral and postcentral gyri, the left insula, and the left paracentral lobule; and the increased component was mainly composed of the striatum structures (bilateral caudate and putamen), the bilateral superior and middle frontal gyri, and occipital regions (Fig. 5B). Furthermore, we found that the mean NBS connectivity strength of the decreased component was negatively correlated with the inattention score in the ADHD patients under multiple thresholds ( $p$ values $<0.05$; Fig. $5 B)$. Collectively, high-resolution network analysis revealed some compatible results of regional (decreased nodal efficiency in the left parietal cortex in ADHD) and NBS analyses compared with lowresolution network analysis. However, several results of global network properties and regional alterations (e.g., increased nodal efficiency in high-resolution analysis) were different. A possible reason for this is that the topological organization of brain networks is largely dependent on spatial scales: a high-resolution network analysis could provide more detailed information about the network changes (Wang et al., 2009b; Zalesky et al., 2010b).

\section{Discussion}

We used probabilistic diffusion tractography and graph theory to investigate the topological organization of the WM networks in drug-naive boys with ADHD and in healthy controls. Although both groups exhibited efficient small-world properties in their WM networks, ADHD patients had decreased global efficiency and increased path length compared with the controls, with the most significant changes were in the left frontal, parietal, and occipital regions. Furthermore, ADHD patients exhibited decreased network connectivity, primarily in the left prefrontal cortex and insula, and increased connectivity, primarily in the medial orbitofrontal cortex and striatum, and these changes were significantly correlated with the inattention and hyperactivity/impulsivity scores of the ADHD group. Together, our study provides empirical evidence for the disruption of topological organization in WM networks in ADHD.

ADHD patients and controls showed small-world properties of the WM networks, characterized by high local clustering and short path length, which are in accordance with previous WM

A

B

C Table 2.
Hubs in $\mathrm{HC}$
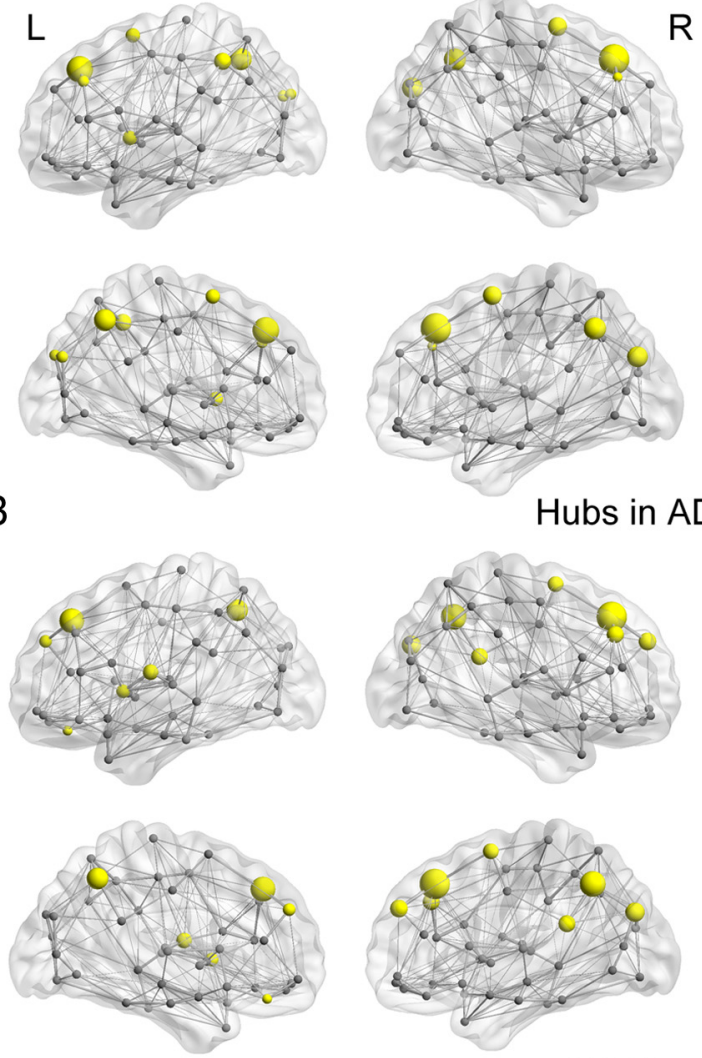

$\mathrm{R}$
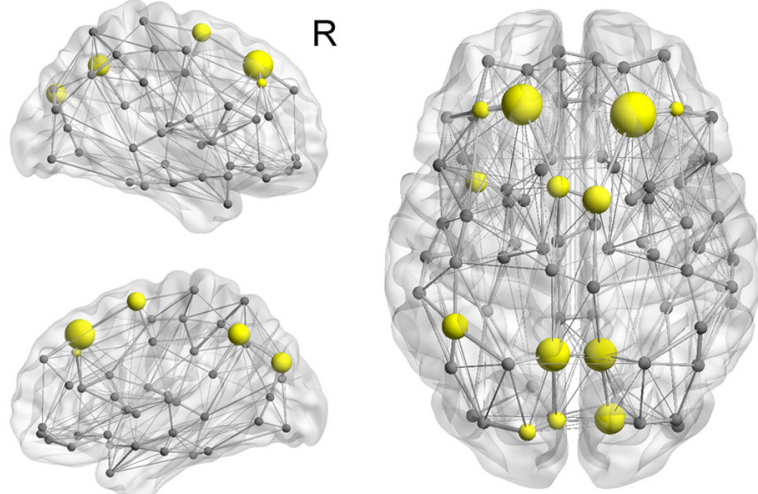

Hubs in ADHD
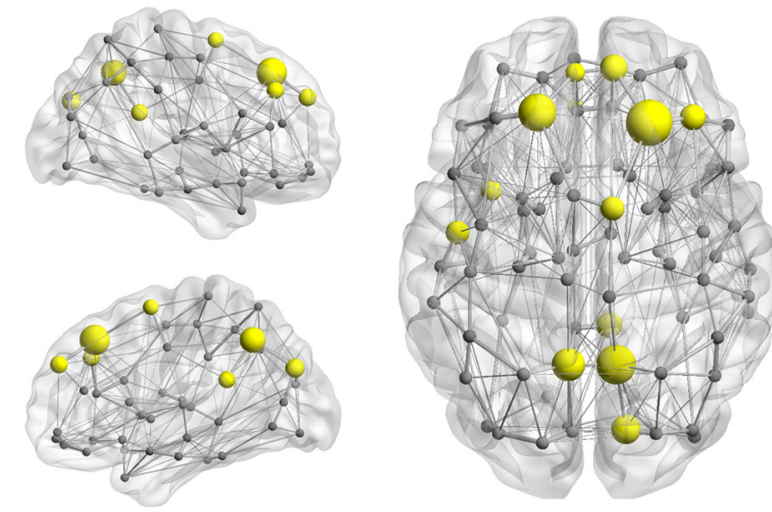

$\mathrm{ADHD}<\mathrm{HC}$
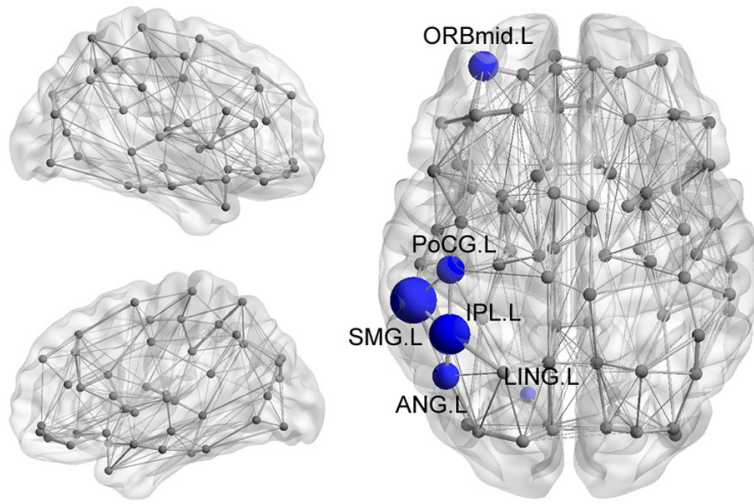

Figure 3. Distribution of hub regions in the WM structural networks of the control and ADHD groups and nodes with decreased efficiency in ADHD patients. $\boldsymbol{A}, \boldsymbol{B}, 3 \mathrm{D}$ representations of the hub distributions in the control $(\boldsymbol{A})$ and $A D H D(\boldsymbol{B})$ groups. The hub nodes are shown in yellow with node sizes indicating their nodal efficiency values. $\boldsymbol{C}$, The disrupted nodes in ADHD patients are shown in blue with node sizes indicating the significance of between-group differences in the regional efficiency. The regions were mapped onto the cortical surface at the axial and sagittal views. Notably, the networks shown here were constructed by averaging the WM connection matrices of all subjects in each group at a sparsity of $10 \%$. The nodal regions are located according to their centroid stereotaxic coordinates. The edge widths represent the connection weights between nodes. The brain graphs were visualized by using BrainNet Viewer software (http://www.nitrc.org/projects/bnv/). HC, Healthy controls. For the abbreviations of nodes, see

network studies in healthy adults (Hagmann et al., 2008; Gong et al., 2009b). Our results suggest that the key aspects of the brain's structural organization are conserved throughout the development process and support the view that small-world networks can tolerate developmental alteration or disease (He et al., 2009; Supekar et al., 2009). Despite the common small-world topology, the ADHD patients showed decreased global efficiency and increased path length. Given that the small-world topology reflects an optimal balance between global integration and local special- 
Table 4. Hub regions of WM networks in control and ADHD groups

\begin{tabular}{|c|c|c|c|c|c|}
\hline \multicolumn{3}{|l|}{ Control } & \multicolumn{3}{|l|}{ ADHD } \\
\hline Hub regions & Class & $E_{\text {nodal }} /$ mean & Hub regions & Class & $E_{\text {nodal }} /$ mean \\
\hline SFGdor.R & Association & 1.13 & SFGdor.R & Association & 1.14 \\
\hline SFGdor.L & Association & 1.12 & PCUN.R & Association & 1.11 \\
\hline PCUN.L & Association & 1.10 & SFGdor.L & Association & 1.11 \\
\hline PCUN.R & Association & 1.10 & PCUN.L & Association & 1.10 \\
\hline CUN.R & Association & 1.10 & CUN.R & Association & 1.09 \\
\hline SMA.R & Association & 1.09 & SFGmed.R & Association & 1.09 \\
\hline IPL.L & Association & 1.09 & MFG.R & Association & 1.08 \\
\hline SMA.L & Association & 1.08 & PCG.R & Paralimbic & 1.08 \\
\hline INS.L & Paralimbic & 1.08 & SMA.R & Association & 1.08 \\
\hline CUN.L & Association & 1.07 & ROL.L & Association & 1.08 \\
\hline MFG.L & Association & 1.07 & INS.L & Paralimbic & 1.07 \\
\hline SOG.L & Association & 1.07 & SFGmed.L & Association & 1.07 \\
\hline MFG.R & Subcortical & 1.06 & REC.L & Paralimbic & 1.06 \\
\hline
\end{tabular}

The hub regions were identified if $E_{\text {nodal }}(i)$ was at least $1 S D$ greater than the mean nodal efficiency of the network $\left[\right.$ i.e., $E_{\text {nodal }}(i)>$ mean $\left.+S D\right]$. The hubs are sorted by the mean normalized nodal efficiency (divided by the mean of all nodes) in each group. The cortical regions are classified as primary, association and paralimbic (Mesulam, 1998). The $E_{\text {nodal }}$ represents the AUC value of the nodal efficiency across thresholds.

Table 5. Brain regions with significant group effects in nodal efficiency

\begin{tabular}{lllll}
\hline & & \multicolumn{2}{l}{$\mathrm{E}_{\text {nodal }}$} & \\
\cline { 3 - 4 } Regions & Category & Control & ADHD & \\
\hline SMG.L & Parietal & $18.18 \pm 0.82$ & $17.53 \pm 0.71$ & 0.011 \\
IPL.L & Parietal & $18.84 \pm 0.91$ & $18.13 \pm 0.90$ & 0.017 \\
ORBmid.L & Frontal & $21.91 \pm 1.02$ & $21.23 \pm 0.94$ & 0.030 \\
PoCG.L & Parietal & $18.60 \pm 1.03$ & $17.95 \pm 0.87$ & 0.032 \\
ANG.L & Parietal & $16.05 \pm 0.81$ & $15.52 \pm 0.81$ & 0.034 \\
LING.L & Occipital & $15.46 \pm 0.92$ & $14.93 \pm 0.79$ & 0.046 \\
\hline
\end{tabular}

Permutation tests were used to determine the differences in nodal efficiency of WM networks between groups ( $p<$ 0.05 , uncorrected) (see Materials and Methods). The values of $E_{\text {nodal }}$ represent the fitted AUC values (mean $\pm S D$ ) of the nodal efficiency of each group.

ization (Sporns et al., 2000), our findings suggest that ADHD children exhibited a less optimized topological organization in their WM networks. The results are supported by a recent R-fMRI study reporting a tendency for reduced global efficiency of brain functional networks in ADHD patients (Wang et al., 2009b). Notably, several studies suggest that the maturation of the healthy human brain follows a "local to distributed" principle (Fair et al., 2009; Dosenbach et al., 2010). Given that the globalefficiency and path-length metrics of the networks are usually associated with integrated information processing, our findings of decreased global efficiency and increased path length in ADHD patients may reflect a delayed structural maturation.

We observed decreased nodal efficiency in ADHD patients, primarily in several frontal (left orbital part of the middle frontal gyrus), parietal (left supramarginal gyrus, left inferior parietal lobe, left postcentral gyrus and left angular gyrus), and occipital regions (left lingual gyrus). These regions are key nodes of the attention networks and exhibit structural and functional abnormalities in ADHD (Bush et al., 2005; Seidman et al., 2005). For instance, several neuroimaging studies have shown decreased gray matter volume or thickness (Hesslinger et al., 2002; Makris et al., 2007), disrupted functional activity (Lee et al., 2005), and disrupted WM integrity (Konrad et al., 2010) in the orbitofrontal region in ADHD. The slower cortical development in this region has also been linked to ADHD (Shaw et al., 2011). Decreased efficiency in the parietal regions is compatible with several fMRI studies that show aberrant activity in the attention system in ADHD (Smith et al., 2006; Tamm et al., 2006; Cao et al., 2008). Specifically, Tamm et al. (2006) reported decreased activation of the left supramarginal and angular gyri in ADHD while perform- ing attention tasks. Previously, we showed decreased activation of the left inferior parietal lobe in ADHD in an alerting task (Cao et al., 2008). The observed ADHD-related decreases in nodal efficiency in the lingual gyrus are largely compatible with our previous R-fMRI work (Wang et al., 2009a). Importantly, we provide structural evidence for the changes that might underlie these functional deficits. Notably, the regions showing ADHD-related decreases in nodal efficiency were located in the left hemisphere. Previous fMRI studies have reported decreased left-hemispheric activity in working memory tasks in ADHD (Valera et al., 2005; Kobel et al., 2009), providing support for our findings. This aberrant laterality could be associated with abnormal brain development in ADHD patients (Cohen et al., 2000).

We observed aberrant structural connectivity in two network components in ADHD patients. The first component with decreased connections was primarily composed of the prefrontal cortex and insular cortex. The prefrontal cortex is mainly involved in attention, motivation, and executive functions (Bush, 2011), and has rich reciprocal interconnections (Durston et al., 2011). Several models of ADHD have emphasized the pivotal role of the prefrontal cortex and its relevant connections in the pathophysiology of ADHD (Barkley, 1997; Sonuga-Barke, 2005; Durston et al., 2011). Numerous diffusion MRI studies have demonstrated abnormal structural connectivity in the prefrontal-related circuitry in ADHD and suggest that a disrupted myelination mechanism underlies the abnormalities (Casey et al., 2007; Pavuluri et al., 2009; Silk et al., 2009). Importantly, we observed a significant correlation between the WM connection strength within the prefrontal component and the inattention score in ADHD patients, suggesting that the aberrant prefrontal-related circuitry is likely to underlie the behavioral symptoms of inattention.

The second component with increased WM connections in ADHD was primarily involved in the medial orbitofrontal regions and striatal structures. Several previous DTI studies have reported increased fractional anisotropy (FA) in frontal WM regions (Silk et al., 2009; Li et al., 2010; Peterson et al., 2011; Tamm et al., 2012) and striatum structures (Peterson et al., 2011; Tamm et al., 2012) in ADHD patients, which provides crucial support for our findings. However, this finding creates a paradox as decreased WM connection probability or FA values are usually associated with disease pathology, Tamm et al. (2012) argued that the atypical increased WM connections in brain disorders might reflect changes in the underlying histology such as fiber diameter, fiber density, extracellular volume fraction, interaxonal spacing, and crossing of fiber pathways. They further argued that increased frontostriatal connections in ADHD might reflect decreased fiber crossing and branching along these pathways. Notably, the result was also compatible with a recent R-fMRI study showing higher functional connectivity in these regions in ADHD (Tomasi and Volkow, 2012). The medial orbitofrontal gyrus and striatum have reciprocal interconnections that subserve the inhibitory functions and reward processing (Barkley, 1997; Durston et al., 2011) and play a crucial role in ADHD pathophysiology (Teicher et al., 2000). Thus, the increased connections of these regions may underlie the symptoms of hyperactivity/impulsivity in ADHD patients.

A recent R-fMRI study reported abnormal network components in a nonclinical, drug-naive sample of high-functioning ADHD (Cocchi et al., 2012). Although several regions comprising the components (e.g., superior frontal gyrus, medial orbitofrontal gyrus and precentral gyrus) partly overlapped with our results, the connections among these regions were different ex- 
A

Group differences in NBS components

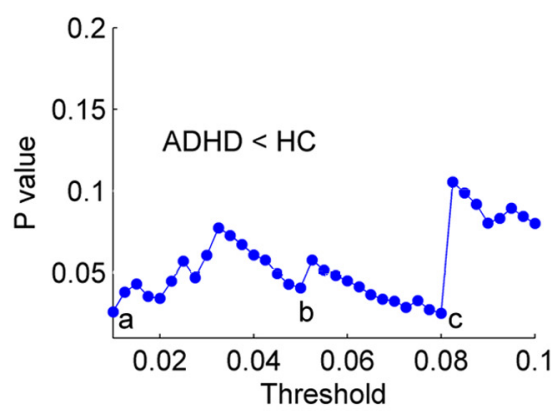

a Threshold $=0.01$

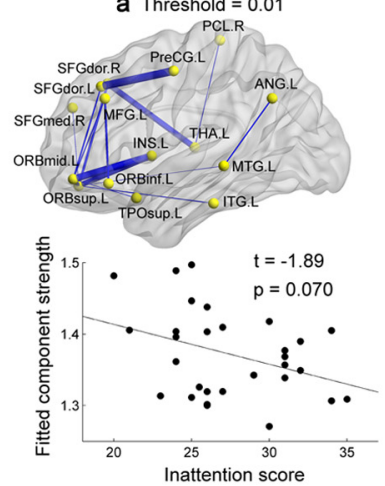

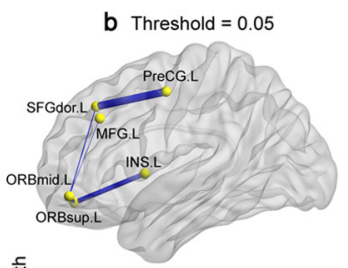

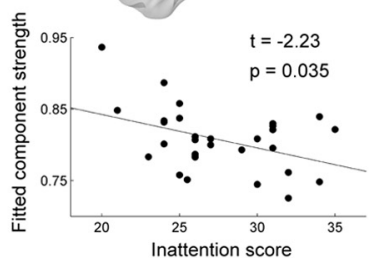

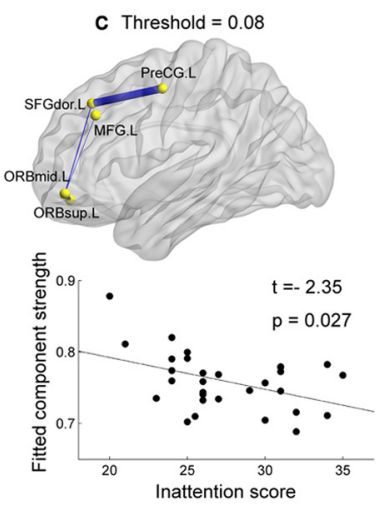

B

Group differences in NBS components
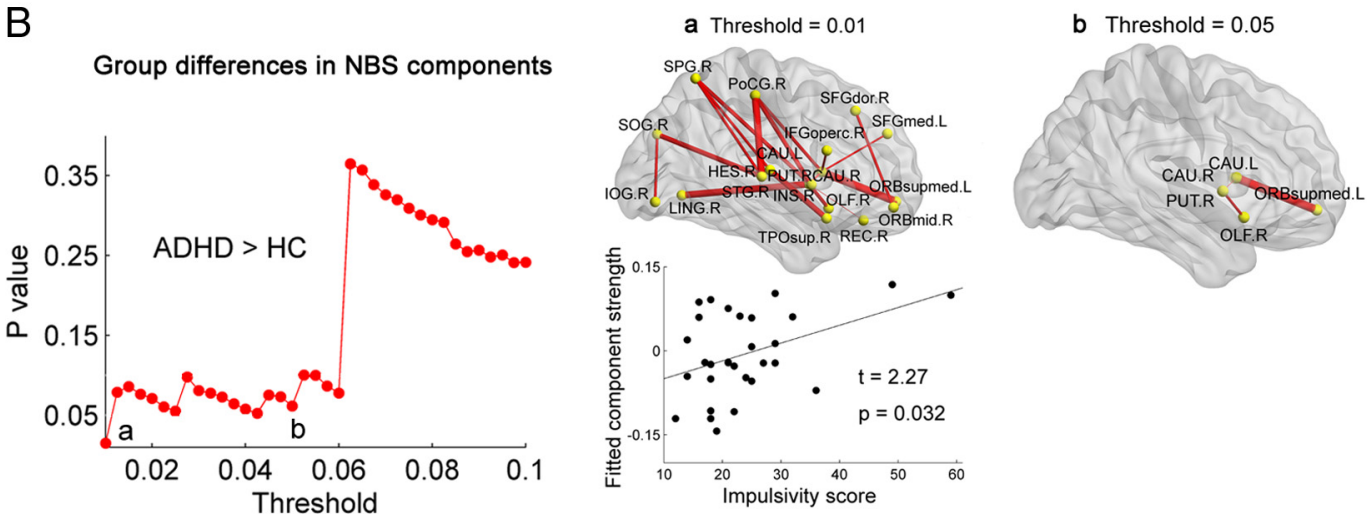

Figure 4. Connected networks that show decreased or increased structural connections in ADHD patients and their relationships with clinical characteristics in patients. $A$, The ADHD patients showed decreased (blue curve) NBS components under a series of probability thresholds (0.01-0.1). The region pairs show decreased structural connections in ADHD patients across several probability thresholds ( $p$ values $<0.05$, corrected). The corresponding sparsities of the network are $24 \%, 10 \%$, and $7 \%$ under the thresholds of 0.01 (a), 0.05 (b), and 0.08 ( $(\boldsymbol{c})$, respectively. These connections formed a single connected network, primarily involving prefrontal and insula regions, and the strength of the component was negatively correlated with the inattention score in ADHD patients. $\boldsymbol{B}$, The ADHD patients showed significantly, or trending toward significantly, increased (red curve) NBS components under a series of probability thresholds (0.01-0.1). The region pairs show increased structural connections in ADHD patients across several probability thresholds ( $p=0.015$ at the threshold of 0.01 and $p=0.061$ at the threshold of 0.05 , corrected) These connections formed a single connected network, primarily involving striatum structures and orbitofrontal regions, and the strength of the component was positively correlated with the impulsivity score in ADHD patients at the threshold of 0.01 (network sparsity $=24 \%$ ). Of note, the correlation was not significant if two outlier points with the highest scores were removed by Shepherd's $\pi$ correlation (Schwarzkopf et al.,2012). In the 3D surface view of the components, the edge widths represent the significance of between-group differences in the connection strength. The nodes and connections were mapped onto the cortical surfaces using BrainNet Viewer software (http://www.nitrc.org/projects/bnv/). For detailed information regarding the WM connections in the significant NBS components, see Table 6. HC, Healthy controls. For the abbreviations of nodes, see Table 2.

Table 6. The strengths, percentages, and frequencies of increased or decreased NBS structural connections across thresholds

\begin{tabular}{|c|c|c|c|c|c|c|c|}
\hline \multicolumn{4}{|l|}{ ADHD < control } & \multicolumn{4}{|l|}{ ADHD > control } \\
\hline Connection & Control & ADHD & Percentage (frequency) & Connection & Control & ADHD & Percentage (frequency) \\
\hline SFGdor.L-PreCG.L & $0.084( \pm 0.015)$ & $0.072( \pm 0.017)$ & $100 \%(20)$ & CAU.L-ORBsupmed.L & $0.028( \pm 0.023)$ & $0.040( \pm 0.024)$ & $100 \%(19)$ \\
\hline SFGdor.L-ORBmid.L & $0.049( \pm 0.019)$ & $0.040( \pm 0.018)$ & $100 \%(20)$ & CAU.R-ORBsupmed.L & $0.020( \pm 0.017)$ & $0.029( \pm 0.018)$ & $100 \%(19)$ \\
\hline MFG.L-ORBmid.L & $0.070( \pm 0.020)$ & $0.062( \pm 0.012)$ & $100 \%(20)$ & CAU.R-PUT.R & $0.063( \pm 0.018)$ & $0.076( \pm 0.017)$ & $100 \%$ (19) \\
\hline ORBsup.L-ORBmid.L & $0.288( \pm 0.035)$ & $0.277( \pm 0.026)$ & $100 \%(20)$ & OLF.R-PUT.R & $0.017( \pm 0.014)$ & $0.026( \pm 0.014)$ & $89 \%(17)$ \\
\hline ORBsup.L-INS.L & $0.034( \pm 0.011)$ & $0.026( \pm 0.009)$ & $50 \%(10)$ & CAU.R-SFGmed.L & $0.013( \pm 0.010)$ & $0.021( \pm 0.014)$ & $74 \%(14)$ \\
\hline ORBmid.LINS.L & $0.015( \pm 0.010)$ & $0.009( \pm 0.006)$ & $35 \%(7)$ & CAU.R-IFGoperc.R & $0.007( \pm 0.006)$ & $0.011( \pm 0.009)$ & $37 \%(7)$ \\
\hline ORBmid.L-TPOsup.L & $0.015( \pm 0.009)$ & $0.011( \pm 0.009)$ & $35 \%(7)$ & REC.R-PUT.R & $0.010( \pm 0.005)$ & $0.013( \pm 0.008)$ & $37 \%(7)$ \\
\hline MFG.L-ORBinf.L & $0.013( \pm 0.006)$ & $0.009( \pm 0.005)$ & $30 \%(6)$ & LING.R-PUT.R & $0.004( \pm 0.003)$ & $0.005( \pm 0.005)$ & $11 \%(2)$ \\
\hline ORBsup.L-MTG.L & $0.010( \pm 0.006)$ & $0.006( \pm 0.005)$ & $25 \%(5)$ & SFGdor.R-ORBmid.R & $0.024( \pm 0.012)$ & $0.032( \pm 0.018)$ & $5 \%(1)$ \\
\hline ANG.L-MTG.L & $0.167( \pm 0.039)$ & $0.150( \pm 0.032)$ & $25 \%(5)$ & ORBmid.R-ORBsupmed.L & $0.003( \pm 0.003)$ & $0.004( \pm 0.005)$ & $5 \%(1)$ \\
\hline ORBsup.L-ITG.L & $0.006( \pm 0.005)$ & $0.004( \pm 0.003)$ & $10 \%(2)$ & INS.R-PoCG.R & $0.024( \pm 0.014)$ & $0.031( \pm 0.016)$ & $5 \%(1)$ \\
\hline PCL.R-THA.R & $0.033( \pm 0.016)$ & $0.030( \pm 0.015)$ & $5 \%(1)$ & INS.R-SPG.R & $0.003( \pm 0.002)$ & $0.005( \pm 0.004)$ & $5 \%(1)$ \\
\hline SFGdor.R-ORBsup.L & $0.004( \pm 0.004)$ & $0.003( \pm 0.002)$ & $5 \%(1)$ & SOG.R-IOG.R & $0.031( \pm 0.021)$ & $0.037( \pm 0.017)$ & $5 \%(1)$ \\
\hline SFGdor.R-THA.R & $0.059( \pm 0.018)$ & $0.049( \pm 0.014)$ & $5 \%(1)$ & SOG.R-STG.R & $0.006( \pm 0.005)$ & $0.010( \pm 0.007)$ & $5 \%(1)$ \\
\hline \multirow[t]{5}{*}{ ORBsup.L-SFGmed.R } & $0.005( \pm 0.004)$ & $0.004( \pm 0.003)$ & $5 \%(1)$ & PoCG.R-CAU.R & $0.003( \pm 0.002)$ & $0.005( \pm 0.004)$ & $5 \%(1)$ \\
\hline & & & & PoCG.R-STG.R & $0.015( \pm 0.005)$ & $0.019( \pm 0.006)$ & $5 \%(1)$ \\
\hline & & & & SPG.R-HES.R & $0.003( \pm 0.004)$ & $0.006( \pm 0.005)$ & $5 \%(1)$ \\
\hline & & & & SPG.R-STG.R & $0.014( \pm 0.008)$ & $0.019( \pm 0.009)$ & $5 \%(1)$ \\
\hline & & & & HES.R-TPOsup.R & $0.007( \pm 0.005)$ & $0.015( \pm 0.011)$ & $5 \%(1)$ \\
\hline
\end{tabular}


A
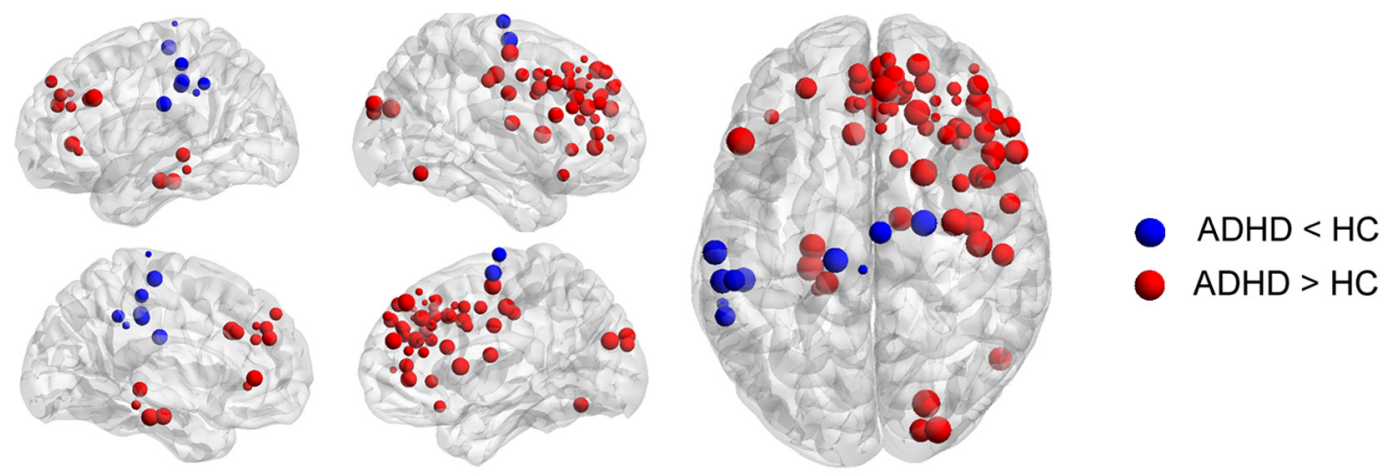

B
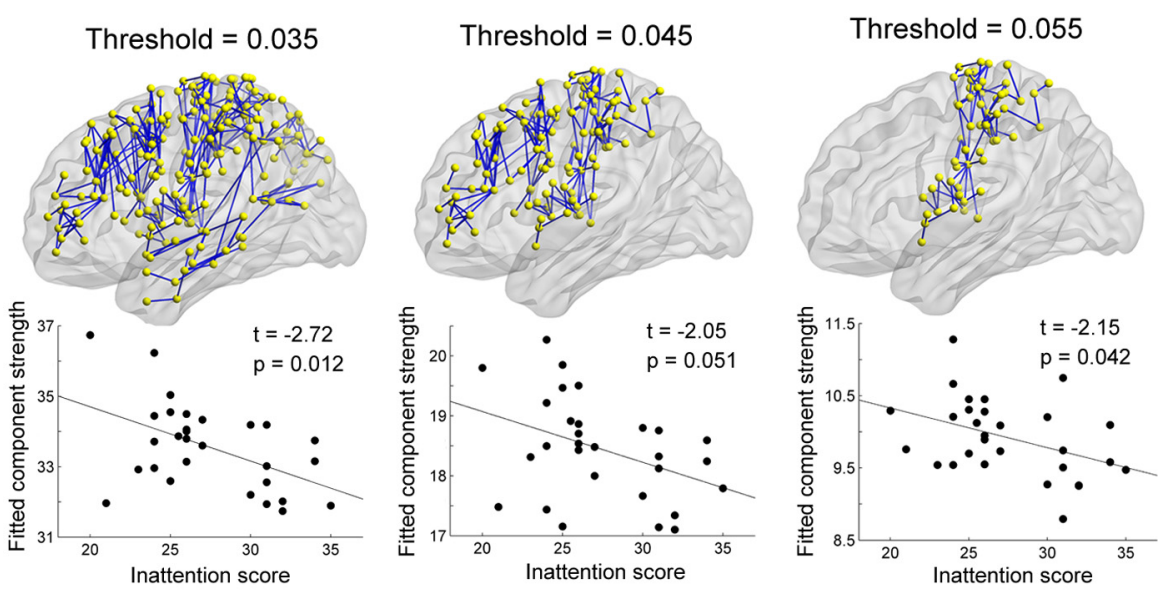

Group differences in NBS components
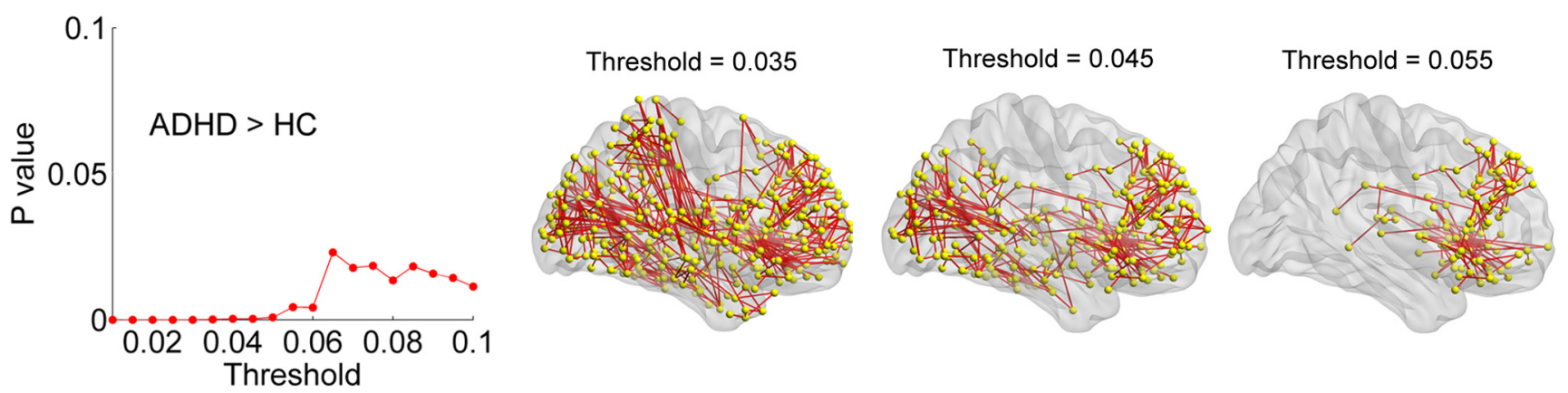

Figure 5. Regional alterations and NBS results in the high-resolution (H-1024) networks. $A$, The nodes with decreased and increased efficiencies in ADHD patients are shown in blue and red, respectively, with node sizes indicating the significance of between-group differences in the regional efficiency. $\boldsymbol{B}$, The ADHD patients showed decreased (blue curve) and increased (red curve) NBS components under a series of probability thresholds (0.01-0.1). Top, The region pairs show decreased structural connections in ADHD patients across several probability thresholds ( $p s<0.05$, corrected). These connections formed a single connected network, primarily involving the left dorsal prefrontal cortex, the left precentral and postcentral gyri, the left insula and the left paracentral lobule, and the strength of the component was negatively correlated with the inattention score in ADHD patients. Bottom, the region pairs show increased structural connections in ADHD patients across several probability thresholds ( $p$ values $<0.05$, corrected). These connections formed a single connected network, primarily involving the striatum structures, the bilateral superior and middle frontal gyri, and occipital regions. The corresponding sparsities of the network are $3.8 \%, 3.1 \%$, and $2.7 \%$ under the probability thresholds of $0.035,0.045$, and 0.055 , respectively. $\mathrm{HC}$, Healthy controls.

cept for decreased orbitofrontal-temporal connectivity. These discrepancies could be due to different imaging modalities (fMRI vs DTI) and different subject characteristics (e.g., age and gender) in the two studies.

Several issues need to be addressed. First, we used the probabilistic tractography method to map whole-brain WM connectivity, which has advantages in tracking specific WM tracts relating to fiber crossing compared with deterministic tractography methods (Behrens et al., 2007). However, such a probabilitybased approach could introduce spurious WM connections that are biologically not connected. The use of a wide probability threshold partly overcame this issue and suggested that our re- sults were not dependent on an arbitrarily chosen threshold. Second, the whole-brain network studies based on MRI data could be sensitive to motion artifacts, especially for the drug-naive ADHD patients. We took several steps to reduce these effects, which included the use of a strap and foam pad during the scan, visually checking the quality of raw images and the head-motion correction of DTI data. However, the potential effects of imaging artifacts still need to be further evaluated. Third, the results for regional efficiency were not corrected for multiple comparisons; therefore, they should only be considered an exploratory analysis. Nonetheless, the results of reduced nodal efficiency in the left parietal cortex in ADHD were highly consistent from different 
templates and different network resolutions. To increase statistical power, future studies need to be conducted using a large sample of ADHD patients. Fourth, we used diffusion MRI data to construct whole-brain WM networks. The brain networks can also be studied using both structural and functional MRI data (Achard et al., 2006; He et al., 2007). The combination of these multimodal neuroimaging techniques would increase our understanding of how structural disruptions in neuronal circuits are associated with functional deficits in ADHD patients. Finally, we used the nonparametric permutation approach as the basis of the network analysis; this method is relatively insensitive to the outliers and does not require the traditional assumption of normal distribution. However, the nonparametric statistical inferences are not generalizable to population-level conclusions about ADHD. Moreover, this study only included boys with ADHD; including girls with ADHD in future studies is important to explore gender effects on the brain networks.

\section{References}

Achard S, Bullmore E (2007) Efficiency and cost of economical brain functional networks. PLoS Comput Biol 3:e17. CrossRef Medline

Achard S, Salvador R, Whitcher B, Suckling J, Bullmore E (2006) A resilient, low-frequency, small-world human brain functional network with highly connected association cortical hubs. J Neurosci 26:63-72. CrossRef Medline

Ashtari M, Kumra S, Bhaskar SL, Clarke T, Thaden E, Cervellione KL, Rhinewine J, Kane JM, Adesman A, Milanaik R, Maytal J, Diamond A, Szeszko P, Ardekani BA (2005) Attention-deficit/hyperactivity disorder: a preliminary diffusion tensor imaging study. Biol Psychiatry 57:448-455. CrossRef Medline

Bai F, Shu N, Yuan Y, Shi Y, Yu H, Wu D, Wang J, Xia M, He Y, Zhang Z (2012) Topologically convergent and divergent structural connectivity patterns between patients with remitted geriatric depression and amnestic mild cognitive impairment. J Neurosci 32:4307-4318. CrossRef Medline

Barkley RA (1997) Behavioral inhibition, sustained attention, and executive functions: constructing a unifying theory of ADHD. Psychol Bull 121:65-94. CrossRef Medline

Basser PJ, Pajevic S, Pierpaoli C, Duda J, Aldroubi A (2000) In vivo fiber tractography using DT-MRI data. Magn Reson Med 44:625-632. CrossRef Medline

Behrens TE, Woolrich MW, Jenkinson M, Johansen-Berg H, Nunes RG, Clare S, Matthews PM, Brady JM, Smith SM (2003) Characterization and propagation of uncertainty in diffusion-weighted MR imaging. Magn Reson Med 50:1077-1088. CrossRef Medline

Behrens TE, Berg HJ, Jbabdi S, Rushworth MF, Woolrich MW (2007) Probabilistic diffusion tractography with multiple fibre orientations: what can we gain? Neuroimage 34:144-155. CrossRef Medline

Bush G (2011) Cingulate, frontal, and parietal cortical dysfunction in attention-deficit/hyperactivity disorder. Biol Psychiatry 69:1160-1167. CrossRef Medline

Bush G, Valera EM, Seidman LJ (2005) Functional neuroimaging of attention-deficit/hyperactivity disorder: a review and suggested future directions. Biol Psychiatry 57:1273-1284. CrossRef Medline

Bush G, Spencer TJ, Holmes J, Shin LM, Valera EM, Seidman LJ, Makris N, Surman C, Aleardi M, Mick E, Biederman J (2008) Functional magnetic resonance imaging of methylphenidate and placebo in attention-deficit/ hyperactivity disorder during the multi-source interference task. Arch Gen Psychiatry 65:102-114. CrossRef Medline

Cao Q, Zang Y, Zhu C, Cao X, Sun L, Zhou X, Wang Y (2008) Alerting deficits in children with attention deficit/hyperactivity disorder: eventrelated fMRI evidence. Brain Res 1219:159-168. CrossRef Medline

Casey BJ, Epstein JN, Buhle J, Liston C, Davidson MC, Tonev ST, Spicer J, Niogi S, Millner AJ, Reiss A, Garrett A, Hinshaw SP, Greenhill LL, Shafritz KM, Vitolo A, Kotler LA, Jarrett MA, Glover G (2007) Frontostriatal connectivity and its role in cognitive control in parent-child dyads with ADHD. Am J Psychiatry 164:1729-1736. CrossRef Medline

Cocchi L, Bramati IE, Zalesky A, Furukawa E, Fontenelle LF, Moll J, Tripp G, Mattos P (2012) Altered functional brain connectivity in a non-clinical sample of young adults with attention-deficit/hyperactivity disorder. J Neurosci 32:17753-17761. CrossRef Medline
Cohen NJ, Vallance DD, Barwick M, Im N, Menna R, Horodezky NB, Isaacson L (2000) The interface between ADHD and language impairment: an examination of language, achievement, and cognitive processing. J Child Psychol Psychiatry 41:353-362. CrossRef Medline

Dosenbach NU, Nardos B, Cohen AL, Fair DA, Power JD, Church JA, Nelson SM, Wig GS, Vogel AC, Lessov-Schlaggar CN, Barnes KA, Dubis JW, Feczko E, Coalson RS, Pruett JR Jr, Barch DM, Petersen SE, Schlaggar BL (2010) Prediction of individual brain maturity using fMRI. Science 329: 1358-1361. CrossRef Medline

DuPaul G, Power TJ, Anastopoulos AD, Reid R, eds (1998) ADHD rating scale-IV: checklists, norms, and clinical interpretations. New York: Guilford.

Durston S, van Belle J, de Zeeuw P (2011) Differentiating frontostriatal and fronto-cerebellar circuits in attention-deficit/hyperactivity disorder. Biol Psychiatry 69:1178-1184. CrossRef Medline

Fair DA, Cohen AL, Power JD, Dosenbach NU, Church JA, Miezin FM, Schlaggar BL, Petersen SE (2009) Functional brain networks develop from a "local to distributed" organization. PLoS Comput Biol 5:e1000381. CrossRef Medline

Fonov V, Evans AC, Botteron K, Almli CR, McKinstry RC, Collins DL (2011) Unbiased average age-appropriate atlases for pediatric studies. Neuroimage 54:313-327. CrossRef Medline

Gong G, Rosa-Neto P, Carbonell F, Chen ZJ, He Y, Evans AC (2009a) Ageand gender-related differences in the cortical anatomical network. J Neurosci 29:15684-15693. CrossRef Medline

Gong G, He Y, Concha L, Lebel C, Gross DW, Evans AC, Beaulieu C (2009b) Mapping anatomical connectivity patterns of human cerebral cortex using in vivo diffusion tensor imaging tractography. Cereb Cortex 19:524-536. CrossRef Medline

Gong YX, Cai TS (1993) Manual of Chinese revised Wechsler Intelligence Scale for Children. Changsha, China: Hunan Atlas Publishing House.

Hagmann P, Cammoun L, Gigandet X, Meuli R, Honey CJ, Wedeen VJ, Sporns O (2008) Mapping the structural core of human cerebral cortex. PLoS Biol 6:e159. CrossRef Medline

He Y, Chen ZJ, Evans AC (2007) Small-world anatomical networks in the human brain revealed by cortical thickness from MRI. Cereb Cortex 17: 2407-2419. CrossRef Medline

He Y, Chen Z, Evans A (2008) Structural insights into aberrant topological patterns of large-scale cortical networks in Alzheimer's disease. J Neurosci 28:4756-4766. CrossRef Medline

He Y, Dagher A, Chen Z, Charil A, Zijdenbos A, Worsley K, Evans A (2009) Impaired small-world efficiency in structural cortical networks in multiple sclerosis associated with white matter lesion load. Brain 132:3366-3379. CrossRef Medline

Hesslinger B, Tebartz van Elst L, Thiel T, Haegele K, Hennig J, Ebert D (2002) Frontoorbital volume reductions in adult patients with attention deficit hyperactivity disorder. Neurosci Lett 328:319-321. CrossRef Medline

Kaufman J, Birmaher B, Brent D, Rao U, Flynn C, Moreci P, Williamson D, Ryan N (1997) Schedule for Affective Disorders and Schizophrenia for School-Age Children-Present and Lifetime Version (K-SADS-PL): initial reliability and validity data. J Am Acad Child Adolesc Psychiatry 36:980 988. CrossRef Medline

Kobel M, Bechtel N, Weber P, Specht K, Klarhöfer M, Scheffler K, Opwis K, Penner IK (2009) Effects of methylphenidate on working memory functioning in children with attention deficit/hyperactivity disorder. Eur J Paediatr Neurol 13:516-523. CrossRef Medline

Konrad A, Dielentheis TF, El Masri D, Bayerl M, Fehr C, Gesierich T, Vucurevic G, Stoeter P, Winterer G (2010) Disturbed structural connectivity is related to inattention and impulsivity in adult attention deficit hyperactivity disorder. Eur J Neurosci 31:912-919. CrossRef Medline

Konrad K, Eickhoff SB (2010) Is the ADHD brain wired differently? A review on structural and functional connectivity in attention deficit hyperactivity disorder. Hum Brain Mapp 31:904-916. CrossRef Medline

Latora V, Marchiori M (2001) Efficient behavior of small-world networks. Phys Rev Lett 87:198701. CrossRef Medline

Lee JS, Kim BN, Kang E, Lee DS, Kim YK, Chung JK, Lee MC, Cho SC (2005) Regional cerebral blood flow in children with attention deficit hyperactivity disorder: comparison before and after methylphenidate treatment. Hum Brain Mapp 24:157-164. CrossRef Medline

Li Q, Sun J, Guo L, Zang Y, Feng Z, Huang X, Yang H, Lv Y, Huang M, Gong Q (2010) Increased fractional anisotropy in white matter of the right frontal region in children with attention-deficit/hyperactivity disorder: a 
diffusion tensor imaging study. Neuro Endocrinol Lett 31:747-753. Medline

Li X (1983) The distribution of left and right handedness in Chinese people. Acta Psychol Sin 15:268-275.

Li Y, Liu Y, Li J, Qin W, Li K, Yu C, Jiang T (2009) Brain anatomical network and intelligence. PLoS Comput Biol 5:e1000395. CrossRef Medline

Liston C, Malter Cohen M, Teslovich T, Levenson D, Casey BJ (2011) Atypical prefrontal connectivity in attention-deficit/hyperactivity disorder: pathway to disease or pathological end point? Biol Psychiatry 69:1168-1177. CrossRef Medline

Lo CY, Wang PN, Chou KH, Wang J, He Y, Lin CP (2010) Diffusion tensor tractography reveals abnormal topological organization in structural cortical networks in Alzheimer's disease. J Neurosci 30:16876-16885. CrossRef Medline

Makris N, Biederman J, Valera EM, Bush G, Kaiser J, Kennedy DN, Caviness VS, Faraone SV, Seidman LJ (2007) Cortical thinning of the attention and executive function networks in adults with attention-deficit/hyperactivity disorder. Cereb Cortex 17:1364-1375. CrossRef Medline

Makris N, Buka SL, Biederman J, Papadimitriou GM, Hodge SM, Valera EM, Brown AB, Bush G, Monuteaux MC, Caviness VS, Kennedy DN, Seidman LJ (2008) Attention and executive systems abnormalities in adults with childhood ADHD: a DT-MRI study of connections. Cereb Cortex 18:1210-1220. CrossRef Medline

Maslov S, Sneppen K (2002) Specificity and stability in topology of protein networks. Science 296:910-913. CrossRef Medline

Mesulam MM (1998) From sensation to cognition. Brain 121:1013-1052. CrossRef Medline

Onnela JP, Saramäki J, Kertész J, Kaski K (2005) Intensity and coherence of motifs in weighted complex networks. Phys Rev E Stat Nonlin Soft Matter Phys 71:065103. CrossRef Medline

Pavuluri MN, Yang S, Kamineni K, Passarotti AM, Srinivasan G, Harral EM, Sweeney JA, Zhou XJ (2009) Diffusion tensor imaging study of white matter fiber tracts in pediatric bipolar disorder and attention-deficit/hyperactivity disorder. Biol Psychiatry 65:586-593. CrossRef Medline

Peterson DJ, Ryan M, Rimrodt SL, Cutting LE, Denckla MB, Kaufmann WE, Mahone EM (2011) Increased regional fractional anisotropy in highly screened attention-deficit hyperactivity disorder (ADHD). J Child Neurol 26:1296-1302. CrossRef Medline

Rubinov M, Sporns O (2010) Complex network measures of brain connectivity: uses and interpretations. Neuroimage 52:1059-1069. CrossRef Medline

Schwarzkopf DS, De Haas B, Rees G (2012) Better ways to improve standards in brain-behavior correlation analysis. Front Hum Neurosci 6:200. CrossRef Medline

Seidman LJ, Valera EM, Makris N (2005) Structural brain imaging of attention-deficit/hyperactivity disorder. Biol Psychiatry 57:1263-1272. CrossRef Medline

Shaw P, Sharp WS, Morrison M, Eckstrand K, Greenstein DK, Clasen LS, Evans AC, Rapoport JL (2009) Psychostimulant treatment and the developing cortex in attention deficit hyperactivity disorder. Am J Psychiatry 166:58-63. CrossRef Medline

Shaw P, Gilliam M, Liverpool M, Weddle C, Malek M, Sharp W, Greenstein D, Evans A, Rapoport J, Giedd J (2011) Cortical development in typically developing children with symptoms of hyperactivity and impulsivity: support for a dimensional view of attention deficit hyperactivity disorder. Am J Psychiatry 168:143-151. CrossRef Medline

Shu N, Liu Y, Li K, Duan Y, Wang J, Yu C, Dong H, Ye J, He Y (2011) Diffusion tensor tractography reveals disrupted topological efficiency in white matter structural networks in multiple sclerosis. Cereb Cortex 21: 2565-2577. CrossRef Medline

Silk TJ, Vance A, Rinehart N, Bradshaw JL, Cunnington R (2009) Whitematter abnormalities in attention deficit hyperactivity disorder: a diffusion tensor imaging study. Hum Brain Mapp 30:2757-2765. CrossRef Medline

Smith AB, Taylor E, Brammer M, Toone B, Rubia K (2006) Task-specific hypoactivation in prefrontal and temporoparietal brain regions during motor inhibition and task switching in medication-naive children and adolescents with attention deficit hyperactivity disorder. Am J Psychiatry 163:1044-1051. CrossRef Medline

Sonuga-Barke EJ (2005) Causal models of attention-deficit/hyperactivity disorder: from common simple deficits to multiple developmental pathways. Biol Psychiatry 57:1231-1238. CrossRef Medline

Sporns O, Tononi G, Edelman GM (2000) Theoretical neuroanatomy: relating anatomical and functional connectivity in graphs and cortical connection matrices. Cereb Cortex 10:127-141. CrossRef Medline

Supekar K, Musen M, Menon V (2009) Development of large-scale functional brain networks in children. PLoS Biol 7:e1000157. CrossRef Medline

Tamm L, Menon V, Reiss AL (2006) Parietal attentional system aberrations during target detection in adolescents with attention deficit hyperactivity disorder: event-related fMRI evidence. Am J Psychiatry 163:1033-1043. CrossRef Medline

Tamm L, Barnea-Goraly N, Reiss AL (2012) Diffusion tensor imaging reveals white matter abnormalities in attention-deficit/hyperactivity disorder. Psychiatry Res 202:150-154. CrossRef Medline

Teicher MH, Anderson CM, Polcari A, Glod CA, Maas LC, Renshaw PF (2000) Functional deficits in basal ganglia of children with attentiondeficit/hyperactivity disorder shown with functional magnetic resonance imaging relaxometry. Nat Med 6:470-473. CrossRef Medline

Tomasi D, Volkow ND (2012) Abnormal functional connectivity in children with attention-deficit/hyperactivity disorder. Biol Psychiatry 71: 443-450. CrossRef Medline

Tzourio-Mazoyer N, Landeau B, Papathanassiou D, Crivello F, Etard O, Delcroix N, Mazoyer B, Joliot M (2002) Automated anatomical labeling of activations in SPM using a macroscopic anatomical parcellation of the MNI MRI single-subject brain. Neuroimage 15:273-289. CrossRef Medline

Valera EM, Faraone SV, Biederman J, Poldrack RA, Seidman LJ (2005) Functional neuroanatomy of working memory in adults with attentiondeficit/hyperactivity disorder. Biol Psychiatry 57:439-447. CrossRef Medline

van den Heuvel MP, Sporns O (2011) Rich-club organization of the human connectome. J Neurosci 31:15775-15786. CrossRef Medline

van den Heuvel MP, Mandl RC, Stam CJ, Kahn RS, Hulshoff Pol HE (2010) Aberrant frontal and temporal complex network structure in schizophrenia: a graph theoretical analysis. J Neurosci 30:15915-15926. CrossRef Medline

Wang L, Zhu C, He Y, Zang Y, Cao Q, Zhang H, Zhong Q, Wang Y (2009a) Altered small-world brain functional networks in children with attentiondeficit/hyperactivity disorder. Hum Brain Mapp 30:638-649. CrossRef Medline

Wang J, Wang L, Zang Y, Yang H, Tang H, Gong Q, Chen Z, Zhu C, He Y (2009b) Parcellation-dependent small-world brain functional networks: a resting-state fMRI study. Hum Brain Mapp 30:1511-1523. CrossRef Medline

Watts DJ, Strogatz SH (1998) Collective dynamics of 'small-world' networks. Nature 393:440-442. CrossRef Medline

Yan C, Gong G, Wang J, Wang D, Liu D, Zhu C, Chen ZJ, Evans A, Zang Y, He Y (2011) Sex- and brain size-related small-world structural cortical networks in young adults: a DTI tractography study. Cereb Cortex 21:449458. CrossRef Medline

Zalesky A, Fornito A, Bullmore ET (2010a) Network-based statistic: identifying differences in brain networks. Neuroimage 53:1197-1207. CrossRef Medline

Zalesky A, Fornito A, Harding IH, Cocchi L, Yücel M, Pantelis C, Bullmore ET (2010b) Whole-brain anatomical networks: does the choice of nodes matter? Neuroimage 50:970-983. CrossRef Medline

Zalesky A, Fornito A, Seal ML, Cocchi L, Westin CF, Bullmore ET, Egan GF, Pantelis C (2011) Disrupted axonal fiber connectivity in schizophrenia. Biol Psychiatry 69:80-89. CrossRef Medline

Zhang J, Wang J, Wu Q, Kuang W, Huang X, He Y, Gong Q (2011) Disrupted brain connectivity networks in drug-naive, first-episode major depressive disorder. Biol Psychiatry 70:334-342. CrossRef Medline 